\title{
Jugend, Freizeit, Medien und Kultur
}

Alles ist anders als früher, widerspruchsvoller. Das »Frottee Trockenschampoo Zeitalter « scheint endgültig vorbei. Die Zeiten, als die »hellblaue Dose (...) noch einen dramatischen Vorfall von Nachfetten der Haare « während eines Rockkonzertes »auf irgendeiner Kuhwiese verhindern musste« (Dörrie 2001), sind vergessen. Auf modernen Musik-Events wird sie nicht mehr benötigt. »Im Kopf ganz frei« schmuggelt man sich aktuell in irgendeine Chefetage ein, wo »OutKast ein kurzes Konzert für die (...) Business-Partner geben«, träumt mit der Band vom utopischen »Stankonia « und lässt sich von einem »distinguierten französischen Barmann« bedienen (Braddock/Hertel 2001). »Frottee« ist hier überflüssig. Heute ist eben alles ist anders als früher. Der »Punker« spielt »Soul-Techno « und der »Gangster-Rapper« Golf. Trotzdem scheint sich nur wenig verändert zu haben. Peter Maffey geht nur noch selten über »sieben Brücken«, dennoch erklimmt er im Februar 2001 Platz eins der MTV-Zuhörer-Charts. »Abraxas « von Santana ist zwar nur noch nostalgieverhangegen Alt-Siebzigern ein Begriff, aber als »Latin-Musiker« hochaktuell - und die Beatles thronen zu Beginn des 21. Jahrhunderts wie vor 35 Jahren an der Spitze der nationalen, europäischen und us-amerikanischen Hitliste (vgl. musikexpress 2001; Juice 2001). Der Beitrag ${ }^{1}$ geht diesen ambivalenten Entwicklungen nach und erkundigt sich, ob und wenn ja inwieweit sich jugendliche Freizeitorientierungen und -formen in den letzten Jahrzehnten modifizierten und welche gesellschaftlichen Entwicklungen die zu beobachtenden Prozesse beeinflussten.

Um den ins Gerede gekommenen, aber dennoch nach wie vor diskutierten theoretischen Positionierungen von Jugend Rechnung zu tragen, werden in einer ersten Annäherung relevante Theoriezugänge in gebotener Kürze vorgestellt und diskutiert (1.). Der Ausdifferenzierung der Ansätze folgend, werden zuerst allgemeine theoretische Ortbestimmungen erörtert (1.1), bevor in einem zweiten Zugriff medientheoretisch akzentuierte Erklärungsansätze ins Zentrum rücken (1.2). Nach einem kurzen, einleitenden Rekurs werden die zeitlichen und materiellen Ressourcenlagen Jugendlicher (2.1) sowie die Ergebnisse der aktuelleren Freizeit- und Medienforschung erörtert (2.2). Auf Grund der enorm angewachsenen Forschungslage ist es angebracht und notwendig, einzelne Ergebnisse der Medienforschung ebenso gesondert zu präsentieren (2.3) wie das Wissen über die Einbindung Jugendlicher in informelle und formelle Gleichaltrigengruppen (2.4). Einem resümierenden

1 Für den unprätentiösen Verzicht auf die Urheberschaft einiger nicht überarbeiteter Passagen des in der zweiten Auflage dieses Handbuchs gemeinsam publizierten Beitrages »Jugend, Freizeit, Medien« danke ich Heinz-Hermann Krüger sowie für die kritische Durchsicht dieser Neufassung ihn und Ernst-Uwe Küster. 
Blick auf die Gesamtpalette der dargestellten Befunde zu jugendlichen Freizeitorientierungen (3.1) folgt abschließend die Nennung der Desiderate, die die bisherige Jugendfreizeitund -medienforschung aufweist, und eine Diskussion der sich daraus ergebenden theoretischen und methodologischen Herausforderungen für die zukünftige Forschung (3.3).

\section{Theoretische Deutungen und Zugriffe}

Die allgemeinen Konzepte und Positionen Entwürfe $\mathrm{zu}$ generativen Unterschieden und Profilen allgemein wie auch die zu den kulturellen und freizeitorientierten Verhaltensweisen von Jugendlichen sind in ihrer Theoriefokussierung uneinheitlicher denn je zuvor. Viele empirische Studien verzichten inzwischen auf eine explizite theoretische Ortsbestimmung ihrer Analysen oder platzieren diese nur noch in Randbemerkungen. Gleichwohl, und in den einleitenden Bemerkungen wurde schon darauf verwiesen, kann hierüber natürlich nicht auf eine durchgehende Theorieabstinenz geschlossen werden, denn zumindest zwischen den Zeilen schimmern die jeweils favorisierten Deutungskonzepte mehr oder weniger durch und spätestens wenn in den Studien die Ergebnisse hinsichtlich ihrer Konsequenzen für pädagogische Handlungskontexte angefragt werden, werfen die gängigen jugendtheoretischen Perspektiven in den unterbreiteten Praxisempfehlungen ihre Schatten. Quasi aus ihren handlungsbezogenen und empirieverhafteten Kontext gelöst, werden nachfolgend einige dieser »Schatten « referiert und kritisch diskutiert. Aus Umfangsgründen muss jedoch durchgängig darauf verzichtet werden, die Einzelnen kontextualen Begründungen und historischen Entwicklungslinien der theoretischen Zugriffe breit zu entfalten.

\subsection{Jugend- und freizeittheoretische Ortsbestimmungen}

\section{Klassische Theorien}

Ein zentraler theoretischer Erklärungsansatz, der sich mit jugendlichen Freizeitkulturen beschäftigt, ist das strukturfunktionalistische Konzept, wie es von T. Parsons und S. N. Eisenstadt entwickelt und in der Bundesrepublik Deutschland in den sechziger Jahren von F. H. Tenbruck (1962) rezipiert und bekannt gemacht worden ist. Strukturfunktionalistische Ortsbestimmungen argumentieren auf der Basis der Existenz einer einheitlichen jugendlichen Teilkultur und begreifen die Jugendkultur als funktional notwendige, relativ eigenständige »interlinking sphere« im Freizeitbereich, die den Jugendlichen die Möglichkeit eröffnet, sich von den partikularistischen Werten der Herkunftsfamilie zu lösen und sich allmählich in die universalistischen Rollenerwartungen der Erwachsenengesellschaft einzuüben. Aus heutiger Perspektive erweist sich diese konzeptionelle Grundnahme für die Analyse jugendlicher Freizeitszenen als wenig geeignet, die zu beobachtenden Ausdifferenzierungs- und Pluralisierungsprozesse jugendkultureller Szenen und Freizeitwelten theoretisch zu erklären. Zudem verschwimmen seit einigen Jahrzehnten die klaren Abgrenzungen von jugendlicher Teilkultur und Erwachsenenkultur einerseits und von kindlichen Kulturen anderseits derart, dass eher von einer Verflüssigung der Generationsgrenzen und der kulturellen Lebensstile auszugehen ist. 
Ein Zweiter, inzwischen zu einem Klassiker in der Jugendfreizeit- und Jugendkulturforschung avancierter Theorieansatz ist der kulturtheoretische Ansatz des Birminghamer Centre for Contemporary Cultural Studies von Clarke u. a. (1979). Die englischen Jugendforscher versuchen die Entstehung jugendlicher Alltagskulturen im Kontext klassenspezifischer Herkunftskulturen zu verorten und das Verhältnis von jugendlichen Sub- und Gegenkulturen und industriell erzeugten Jugendkulturen als dialektisches Spannungsverhältnis zu interpretieren. Die hegemoniale Jugendfreizeitkultur liefert den Subkulturen einerseits das kulturelle Rohmaterial, aus dem diese oppositionelle Bedeutungen herausschlagen. Dieser Ortung folgend werden jugendliche Stilbastler nicht als manipulierte Opfer eines allgegenwärtigen Konsumzwanges gesehen, sondern als aktive und kreative Träger kulturellen und gesellschaftlichen Wandelns. Andererseits gelingt es jedoch den industriell erzeugten Jugendkulturen, die subkulturellen Gruppenstile zu reintegrieren, indem sie die oppositionellen stilistischen Neuerungen aufgreifen, entschärfen und als modische Innovationen neu auf den Markt bringen. Der kulturtheoretische Ansatz des CCCS wurde insbesondere in den 80er-Jahren des letzten Jahrhunderts in der westdeutschen Kinder- und Jugendkulturforschung breit rezipiert und zum Bezugspunkt einer Reihe von empirischen Jugendkulturstudien (vgl. zusammenfassend Baacke/Ferchhoff 1992). Ein Transfer der theoretischen Grundannahmen dieses Ansatzes auf die westdeutschen Verhältnisse war damals und ist heute jedoch nur begrenzt möglich. Denn im Unterschied zu den Entwicklungen und Forschungen in Großbritannien entwickeln sich in der Bundesrepublik Deutschland die Jugendsubkulturen nicht mehr deckungsgleich zu den existierenden Klassen- und Schichtstrukturen. Angesichts erheblicher Auflösungserscheinungen traditioneller Sozialmilieus und kultureller Klassenbindungen ist es keineswegs durchgängig möglich, jugendliche Subkulturen präzise in klassenspezifischen Stammkulturen zu lokalisieren. Das implizier allerdings nicht, dass die Herausbildung jugendlicher Freizeitszenen und -gruppen inzwischen vollends beliebig ist. Auch heute noch wird sie durch Soziallage und Bildungsniveau wesentlich mit beeinflusst. Hierauf hingewiesen zu haben, ist ein Verdienst der klassenund kulturtheoretischen Ortsbestimmungen. Auch die über eine kritische Durchsicht individualisierungstheoretischer Diagnosen herausfiltrierte Erkenntnis, dass heutige »Jugendkulturen in eigenwilliger, eben expressiver Form die neuen sozialen Ungleichheiten in der Gesellschaft repräsentieren und sichtbar machen«(Wensierski 2000, S. 210), verdankt sich nicht unwesentlich diesem Theoriekonzept.

In den sozialwissenschaftlichen Jugendtheorien der letzten Jahrzehnte wurde zuweilen ignoriert, dass ein unmittelbarer Zusammenhang zwischen den physischen und psychischen Entwicklungen im Jugendalter besteht. Auf dieses Defizit weisen neuerdings entwicklungspsychologische Theorieperspektiven wieder verstärkt hin. Im Kontrast zur älteren Entwicklungspychologie akzentuieren die aktuellen Vorschläge jedoch stärker gesellschafts- und sozialwissenschaftliche Theorie- und Empiriebestände und betonen ihre Distanz zu starren, phasenbezogenen entwicklungspsychologischen Konzeptionen. Stattdessen wenden sie sich lebenslauf- und biographieorientierten Analyse- und Theoriemodellen zu, die kultur- und freizeitbezogene Aspekte zu beachten eher zulassen als stadienfixierte Modellkonstruktionen (vgl. u. a. Wiesner/Silbereisen 1996; Fend 2000). 


\section{Lebensweltorientierte und sozialökologische Ansätze}

Lebensweltlich und in geringerem Maße auch sozialökologisch angelegte theoretische Positionierungen erlebten insbesondere Ende der 1980er-Jahre eine fast schon als inflationär anzusehende Konjunktur.

Lebensweltliche Ansätze knüpfen grundlegend an die phänomenologisch-interpretative Soziologie an. In Abgrenzung zur normativ gesättigten Empirie der Tatsachenparadigmen favorisieren verstehensorientierte Zugänge eine totalitätsbezogene Empirie und Theorie. Der Symbolische Interaktionismus und die Ethnomethodologie sind neben wissens- und sprachsoziologischen Ansätzen als Ausdifferenzierungen der phänomenologischen (»lebensweltlichen«) Soziologie (vgl. u. a. die Beiträge in Hitzler/Honer 1997) zu verstehen. Die Prämissen einer lebensweltlichen Theorieperspektive formulierte A. Schütz in Anknüpfung an die Phänomenologie E. Husserls. Dem Entwurf liegt die Intention zu Grunde, ein theoretisches Gerüst vorzulegen, welches es ermöglicht, die soziale Welt so zu verstehen wie das subjektive Erkennen diese sich erarbeitet. Ethnomethodologie und Symbolischer Interaktionismus interessiert hieran anschließend (zum theoretischen Programm des Ansatzes vgl. u. a. Waldenfels 1985; Grathoff 1989), welche alltäglichen Strukturen die Gesellschaft zusammenfügen beziehungsweise mit welchen Sinnhorizonten die Alltagsakteure ihre Handlungen unterlegen.

In Beachtung dieser theoretischen Modelle beobachtete die Projektgruppe Jugendbüro $(1973,1977)$ schon vor inzwischen knapp dreißig Jahren die Lebenswelten von HauptschülerInnen. M. Kieper (1980) arbeitete nach einem Rekurs auf die phänomenologischinterpretativen Forschungsansätze die Kompetenzen von »verwahrlosten« jugendlichen Mädchen bei der subjektiven Strukturierung der Lebenswelt heraus. Implizit beleuchten beide Studien auch das Verhalten von Jugendlichen in Familie und Freizeitkultur. Den Zusammenhang von Handlungs- und Sozialräumen und ihre Wirkungen auf soziale Milieus arbeitete die Arbeitsgruppe Jugendforschung mit einer materialistisch unterlegten lebensweltlichen Perspektive heraus (vgl. Becker u. a. 1984). Unterschiedliche Handlungsformen von Jugendlichen in ihrer Freizeit beschrieb K. Lenz (1988). Gegen das Konzept einer einheitlichen Gestalt Jugend argumentierend, schlägt K. Lenz vor, zwischen familien-, hedonistisch-, maskulin- und subjektorientierten jugendlichen Handlungsformen zu unterscheiden. An das kommunikativ reformulierte lebenswelttheoretische Paradigma anknüpfend (vgl. Habermas 1981), aber gleichfalls gegen das Muster einer einheitlichen Lebensphase Jugend argumentierend, interessierte sich W. Thole (1991) für den Alltag einer jugendlichen Szene und ihr Verhalten in institutionalisierten wie ei gengestalteten Handlungsräumen, ihren Umgang miteinander und mit den gesell schaftlichen Normen. Jugendlichen aus vergleichbaren sozialen Milieus wenden sich neuere Studien unter Bezug auf ethnomethodologische Theorie- und Forschungsansätze zu (vgl. u. a. Tertilt 1996; Küster 2001). Unter explizit ausformulierten sozialpädagogischen Fragestellungen untersuchten B. Müller, R. Rosenow und M. Wagner (1994) in einer im Kern lebensweltlichen Studie Dorfkulturen in »Ost « und »West «.

Im Unterschied zu den lebensweltlichen Paradigmen, die sich an phänomenologisch und/oder kommunikativ-intersubjektiv begründete Theoriestränge anlehnen, entfalten sozi- 
alökologische Ansätze ihr Konzept mit Bezug auf feldtheoretisch-raumbezogene und ökopsychologische Theorietraditionen. Das Vorhaben des sozialökologischen Ansatzes, die Interdependenzen zwischen strukturellen und subjektiven Dimensionen offen zu legen (vgl. Bronfenbrenner 1976), fand anfänglich insbesondere in der Schulforschung Berücksichtigung. Aktuell finden sozialökologisch orientierte Ansätze unter dem Stichwort »sozialräumlich « insbesondere in Evaluations- und Konzeptentwicklungsprojekten zur außerschulischen Kinder- und Jugendarbeit Anwendung (vgl. u. a. Deinet 1999). Im Kontext der Jugendforschung untersuchten S. Hübner-Funk u. a. (1983) unter Rückgriff auf sozialökologische Modelle die Beziehungen zwischen den Lebensbedingungen und -orientierungen von Jugendlichen in drei unterschiedlichen Sozialräumen. Den erziehenden und sozialisierenden Einfluss der Wohnumwelt auf das jugendliche Freizeit- und Medienverhalten gingen R. Tippelt u. a. (1986) nach. I. Müller (1984) erforschte Szenarien der Umweltaneignung von Jugendlichen. Beziehen sich die genannten Studien lediglich forschungspragmatisch auf die theoretischen Entwürfe und verzichten darauf, ihre Erkenntnisse theoriegenerierend auszuwerten, belegen schon in den 1980er-Jahren U. Sander und R. Vollbrecht (1985) unter Hinzuziehung des von D. Baacke entwickelten sozialökologischen Theoriekonzepts die empirischen Möglichkeiten des Ansatzes in Bezug auf die Erschließung subjektiver Deutungsmuster.

Lebenswelt- und sozialökologisch orientierte Theorie- und Forschungskonzeptionen haben insbesondere Ende der 1980er und zu Beginn der 1990er-Jahre die Jugendfreizeit- und Jugendmedienforschung produktiv belebt und dazu beigetragen, Jugend mehr als Subjekt und damit sozialer Konstrukteur denn als Objekt von Freizeitwelten und Medienlandschaften $\mathrm{zu}$ verstehen. Die wissenschaftsgenerierende Bedeutung des Ansatzes ist angedeutet, jedoch noch nicht ausgeschöpft. Die teilweise theorieungeleitete Beliebigkeit, mit dem Rekurse auf lebensweltliche Theorietraditionen erfolgen, ist jedoch nicht zu übersehen. Versuche, durch theoretische Ergänzungen die diagnostizierten Schwächen des lebensweltlichen, aber auch des sozialökologischen Theorems zu überwinden und so die Ansätze auch für die Erschließung subjektiver und gesellschaftlicher Strukturen zu öffnen, werden gegenwärtig nicht weiter verfolgt. Verstärkt finden in den letzten Jahren Kernüberlegungen lebens- und sozialökologischer Theoriemodelle in der Jugendforschung insbesondere in ethnographisch angelegten Studien Beachtung und einen theoriegenerierenden Ort (vgl. u. a. Rieker 2000; Zinnecker 2000a; Thole/Müller 2001).

\section{Modernisierungstheoretische Theorieperspektiven und Lebensstilansätze}

Im Kontext der makrosoziologischen Diskussion, die sich mit der Erklärung des Wandels und den gesellschaftlichen Determinanten jugendlicher Freizeit- und Medienwelten beschäftigt, erfahren in den letzten Jahren vor allem zwei Theoriekonzepte eine besondere Aufmerksamkeit: es ist zum einen das Konzept einer kritischen Modernisierungstheorie, wie es vor allem von U. Beck (1983, 1986, 2000) entwickelt worden ist, und zum anderen die Kultursoziologie der Lebensstile als einer Theorie der sozialen Ungleichheit, wie sie von dem französischen Soziologen P. Bourdieu (1984) vorgelegt worden ist und in einer Studie über das alltägliche Leiden an der Gesellschaft eine eindrucksvolle empirische Ü- 
berprüfung mittels einer »realistisch-konstruktiven« Perspektive fand (Bourdieu u.a. 1997).

Die kritische Modernisierungstheorie untersucht und beobachtet die ambivalenten Folgen der wohlfahrtsstaatlichen Modernisierung der bundesrepublikanischen Gesellschaft seit der Nachkriegszeit. Die Verbesserung des materiellen Lebensstandards für alle hat allerdings, so die Diagnose, nicht die Herausbildung neuer und Stabilisierung alter sozialer Ungleichheiten verhindert, jedoch u. a. zu einer Infragestellung geschlechtsständischer Zuweisungen bei gleichzeitiger Konstanz alter Rollenzuweisungen sowie zu einer Auflösung klassenspezifischer Sozialmilieus und einer soziokulturellen Freisetzung aus überlieferten Traditionen geführt.

Das von den ProtagonistInnen der kritischen Modernisierungstheorie präsentierte dominante Deutungsmuster heißt Individualisierung. Mit Individualisierung ist jedoch keinesfalls, wie häufig angenommen, Individuierung oder gar Vereinsamung und Verinselung der Gesellschaftsangehörigen gemeint, sondern die Dynamisierung der sozialen Beziehungsformen und -regeln am Ende des 20. Jahrhunderts mit gravierenden Folgen für die Konstitution des Sozialen und der Subjekte.

Individualisierung ist somit nicht nur der semantische Code für die oft gehuldigte »Biographisierung « bisher standardisierter Lebenslaufmuster, sondern auch und insbesondere Stichwort einer paradoxen Entwicklung der »Herstellung, Selbstgestaltung, Selbstinszenierung nicht nur der eigenen Biographie, sondern auch ihrer Einbindungen und Netzwerke, und dies im Wechsel der Präferenzen und Lebensphasen und unter dauernder Abstimmung mit anderen und den Vorgaben von Arbeitsmarkt, Bildungssystem und Wohlfahrtsstaat « (Beck/Beck-Gernsheim 1994, S. 14). Der Weg durchs Leben gestaltet sich nicht mehr als schablonierter Lebenslauf, sondern als biographische Bricolage.

Unter Bezug auf die modernisierungstheoretische Gesellschaftsdiagnose interpretierten seit Mitte der 1980er-Jahre eine Reihe von AutorInnen (vgl. u. a. Zinnecker 1987; Heitmeyer/Peter 1988; Krüger 1991; Matthesius 1992; Jugendwerk der Deutsche Shell 1997, 2000; Meyer 2000) die empirisch konstatierbaren Veränderungsprozesse im Bereich jugendlicher Freizeit- und Medienwelten. So hat beispielsweise H. H. Krüger (1991) aufgezeigt, dass sich im Bereich des Freizeitvolumens, des finanziellen Freizeitbudgets und der Ausstattung mit Medien die Lebenslagen von Jugendlichen aus unterschiedlichen Bildungsniveaus bzw. sozioökonomischen Statusgruppen zwar angeglichen haben, hinter der vordergründigen Fassade einer Homogenisierung von Freizeitbedingungen und einer Vielfalt von Freizeitmöglichkeiten jedoch alte Ungleichheiten in neuem Gewande fortbestehen. Jugendliche aus niedrigen sozioökonomischen Statusgruppen konsumieren beispielsweise vermehrter als andere Unterhaltungs- und Aktionfilme im Fernsehen oder auf Video und bevorzugen eher praktisch-gegenständlichen Hobbys. Die Heranwachsenden aus den oberen sozialen Statusgruppen bevorzugen hingegen eher die Printmedien, favorisieren ästhetisch-kulturelle Freizeitpraxen und nutzen ihre Freizeit auch zur Weiterbildung. Ähnlich ambivalent stellen sich auch die Veränderungstendenzen im Freizeitbereich für die weiblichen Jugendlichen dar. So haben die Mädchen im Unterschied zu den 50er und 60er-Jahren des letzten Jahrhunderts inzwischen auch den öffentlichen Handlungsraum für sich erobert 
und sind genauso häufig wie die Jungen in die informellen Netzwerke der Gesellschaft der Altersgleichen eingebunden. Neben diesen Tendenzen zur Geschlechterangleichung lassen sich auch traditionelle Muster geschlechtsspezifischer Zuweisung ebenso wie neue Formen geschlechtsspezifischer Diskriminierung aufzeigen (vgl. u. a. Luca 1997; Rose 1997).

Analoge Ambivalenzen stecken auch in der von U. Beck (1986, S. 152) diagnostizierten »Enttraditionalisierung von sozialmoralischen Milieus«, in dem Bedeutungsverlust von Kirchen, Nachbarschaften oder traditionellen Vereinen, an deren Stelle heute eine stärkere Informalisierung von Sozialkontakten auch bei Jugendlichen tritt. Dadurch erhalten die Heranwachsenden zwar die Möglichkeit, sich ihre Freundschaftsnetze selbstständig aufzubauen, gleichzeitig gehen damit jedoch die Sicherheiten traditionsbezogener Bindungen und die Stabilität und Nähe sozialer Milieus verloren (vgl. u. a. Bäumler/Bangert/Schwab 1994; Eckert/Reis/Wetzstein 2000; Wensierski 2000). Auch die Auswahl und Pluralisierung von Medienangeboten - vor allem bedingt durch die Entwicklung der »Neuen Medien« - ist deutlich präformiert durch die strukturellen Modernisierungen.

Neben der kritischen Modernisierungstheorie hat in jüngster Zeit vor allem eine Soziologie der Lebensstile (vgl. Bourdieu 1984) im theoretischen Diskurs der Jugendfreizeitforschung an Bedeutung gewonnen. Lebensstile sind nach P. Bourdieu Ausdruck moderner Klassenverhältnisse in entwickelten Konsumgesellschaften. Die Klassenbasis als strukturelle Grundlage der Entwicklung eines Lebensstils wird jedoch gegenüber der alten Klassentheorie erweitert. Neben das ökonomische Kapital - Einkommen und Vermögen - treten das soziale Kapital und das kulturelle Kapital in der Form der Verfügbarkeit über Kulturgüter sowie als individuelle Kompetenz.

Die Stärke der von P. Bourdieu (1984) entwickelten Kultursoziologie liegt sicherlich darin, dass sie durch die Unterscheidung unterschiedlicher Kapitalressourcen die traditionelle marxistische Klassentheorie erweitert und sie zu einer Theoriefolie entwickelt, die es erlaubt, Veränderungsprozesse jugendlichen Freizeit- und Medienverhaltens im Raum sozialer Klassenfraktionen zu verorten. Der Nachteil dieses gesellschaftstheoretischen Modells ist, dass es sehr statisch ist und Wanderungs- und Assimilierungsbewegungen im Raum sozialer Klassen ebenso wenig fassen kann wie wegen der nur schwach angedeuteten subjekttheoretischen Perspektive die Erosionsprozesse kultureller Identitäten. Zudem zeigt sich die Komplexität dieses Theoriemodell gegenüber empirischen Operationalisierungen sperrig. In einzelnen Studien und Diskussionsbeiträgen sind zwar implizite Bezugnahmen zu erkennen (vgl. Schäffer 1996; Neumann-Braun/Deppermann 1998), eine explizit, sich auf diese theoretische Gegenwartsdiagnose beziehende neuere Studie für den deutschsprachigen Raum in Bezug auf jugendliches Freizeit- und Medienverhalten liegt jedoch nicht vor.

In jüngster Zeit greifen poststrukturalistische Theorieperspektiven (vgl. u. a. Ferchhoff/Neubauer 1997; Hitzler/Bucher/Niederbacher 2001) modernisierungstheoretische Erkenntnisse auf und konkretisieren den Vorschlag, von pauschalisierenden Jugendbildern Abschied zu nehmen. In Distanz jedoch zu vorliegenden Theoriemodellen plädieren sie für Theoriekonzepte, die es ermöglichen, die patchworkartigen Identitätsbildungsprozesse von Jugendlichen wahrzunehmen und die ausdifferenzierte jugendkulturelle Szenerie empirisch 
zu erkennen. Sensibel für die Binnenstruktur insbesondere avantgardistischer Jugendszenen ist ein vergleichbares, allerdings um deutlich herausgestellte ideologie- und kulturkritische Argumente angereichertes Beobachtungsmodell auch bei jüngeren »SzeneforscherInnen« auszumachen (vgl. u. a. Holert/Terkessidis 1996; Zeitschrift »DE:BUG «). Werden die hierüber theoretisch angereicherten Kultur- und Freizeitstudien richtig verstanden, dann plädieren sie implizit für die Substituierung systematischer jugendtheoretischer Ortsbestimmungen durch ein mehr oder weniger theoretisch abgesichertes, keineswegs jedoch eindimensional angelegtes »Kursbuch Jugendkultur« (SPoKK 1997).

\subsection{Medientheoretische und -rezeptive Ortsbestimmungen}

Die gegenwärtig erkennbaren medientheoretischen Konzeptionen zeigen deutliche Überschneidungen mit den allgemeinen jugendtheoretischen und somit auch freizeitorientierten Standortbestimmungen. Gleichwohl sind einige konkretisierende Theorieansätze - in Ergänzung zu den klassischen Imitations-, Kartharsis-, Inhibitions- und Habilitationshypothesen -, in den letzten Jahren in der Medienforschung herausgearbeitet worden (vgl. übersichtlich u. a. Barthelmes/Sander 1997; Paus-Haase 1999; Eberle 2000):

- Medienzentristische Ansätze, die unter den Stichworten »Stimulus-Response-Ansatz« oder der »Kultuvierungshypothese « bekannt wurden, gehen von der Modellvorstellung aus, dass Medien bei den RezipientInnen direkte Wirkungen hervorrufen. Entsprechend codierte Erklärungsansätze und Forschungsperspektiven konzentrieren sich den theoretisch explizierten Vorstellungen nach auch nicht primär auf die MedienkonsumentInnen, sondern primär auf die ästhetischen, formalen, dramaturgischen und inhaltlichen Gestaltungsformen der Medien selbst. Die MediennutzerInnen werden gängigen Studien zufolge allenfalls als Extrem-, Viel-, Wenig- oder NichtkonsumentInnen, nach ihrer Zugehörigkeit zu unterschiedlichen Altersgruppen und in seltenen Fällen allenfalls noch nach ihrer sozialen Lage und ihren Lebensbedingungen differenziert. Insbesondere in der psychologischen Aggressions- und Gewaltforschung bilden Varianten des »StimulusResponse-Ansatzes« anerkannte Grundlagen, obwohl auch hier zuweilen die augenfällige Theoriearmut der zu Grunde gelegten Modelle kritisch angemerkt wird. Gleichwohl werden mithilfe dieses Ansatzes erhobene Daten immer wieder zitiert, um beispielsweise zu belegen, dass der übermäßige Konsum von gewalthaltigen Darstellungen im Fernsehen nachhaltige Folgen für Kinder und Jugendliche hat und diese dazu animiert, ihrerseits Probleme ebenfalls unter Rückgriff auf gewaltförmige, nichtsprachliche Handlungsstrategien zu lösen. Kritisch ist gegenüber diesem medientheoretischen Konzept so insbesondere eine Missachtung der spezifischen, subjektiven Verarbeitungsformen der Medienrepräsentationen durch die RezipientInnen, eine Ignoranz gegenüber den sozialen und kulturellen Situationen der Medienrezeption sowie eine Fixierung auf kausale Interpretationsmuster vorzutragen, die darauf basieren, eindimensional statt multikausal zu interpretieren.

- Auch gesellschafts-, kultur- und ideologiekritische medienbezogene Theorieperspektiven sind in gewisser Weise medienzentristisch akzentuiert, zumindest dann, wenn sie einbahnstraßenähnlich ihre Beobachtungen an massenmedial vermittelte Inhalte und Formen anbinden. Dort allerdings, wo an den frühen Studien der Frankfurter Schule anzuknüpfen versucht wird und den Vergesellschaftungsbedingungen mit Blick auf die industriellen Formen der Produktion von Kultur nachgegangen wird, verliert sich diese enge Perspektive und erweitert sich hin zu den rezipierenden Subjekten, gerät in den Blick, dass es die entfremdeten Lern- und Arbeitsverhältnisse bürgerlicher Gesellschaften selbst sind, die ein Amüsement fernab der eigentlichen Bedürfnisse einfordern (vgl. Horkheimer/Adorno 1969). Neben U. Oevermanns Suche (1983) nach den objektiven Sinnstrukturen der Fernsehinhalte betrat in den letzten Jahren P. Bourdieu (1998) die fernsehkri- 
tische Bühne. Ihm folgend wären es insbesondere ökonomische Zwänge, die die Fernsehinhalte maßgeblich zensieren und Fernsehen zu einer »besonders schädlichen Form symbolischer Gewalt« (Bourdieu 1998, S. 21) und aus dem »Be-schreiben der sozialen Welt durch das Fernsehen ein Vor-schreiben « (Bourdieu 1998, S. 28) machen. P. Bourdieu verklärt hier die gesellschaftliche Wirkung und Bedeutung des Fernsehen insofern, als dass er hinter die kulturkritischen Analysen der Frankfurter Schule wie auch hinter seine eigenen gesellschaftsanalytischen Studien zurückfällt, weil er sowohl den Vergesellschaftungsmodus dieses Mediums wie auch die subjektiven Verarbeitungs- und Motivstrukturen der RezipientInnen unbeobachtet lässt.

- Unter den mediennutzerInnenorientierten Theorieperspektiven kann der »Uses-andGratifications-Ansatz« bis heute als der Kernansatz identifiziert werden. Subjektive Bedürfnisse konstituieren im Zusammenspiel mit sozialen Dispositionen einerseits und gesellschaftlichen Strukturen andererseits spezifische Motive und Handlungsinteressen und diese wiederum spezifische Alltags- und Problemlösungsmuster. Daraus sich ergebene Handlungen motivieren zur Rezeption u. a. von medialen Repräsentationen mit der Intention, die aufgeschichteten Bedürfnisse zu befriedigen. Kaum ausformuliert verbirgt sich damit in diesem Ansatz eine handlungstheoretische Perspektive. Empirisch operationalisiert sich dieses Konzept insbesondere in nutzerInnenzentrierten Medienanalysen, beispielsweise in Studien, die die Abhängigkeit der NutzerInnenverhaltensweisen von den sie umgebenen Lebenssituationen eruieren. Die Validität der auf Grundlage des »Uses-and-Gratifications-Ansatzes« erhobenen Befunde ist allerdings kritisch zu beurteilen. Ihre theoretischen Schwachstellen wurden insbesondere im deutschsprachigen Raum diskutiert und unter Verweis auf kognitionstheoretische und/oder entwicklungspsychologische Erkenntnisse im so genannten »Nutzenansatz« durch Berücksichtigung der objektiven und subjektiven sozialkulturellen Lebenslagen der RezipientInnen und ihrer Motive empirisch validiert. Studien weisen darauf hin, dass Jugendliche Medien auch nutzen, um ihre Alltagserfahrungen angemessen be- und verarbeiten zu können (vgl. Paus-Haase u. a. 1999). Im Kern ebenfalls nutzerInnenorientiert sind konstruktivistische Perspektiven, also Ansätze, die davon ausgehen, dass es dem »Individuum nicht gelingen kann, die Wirklichkeit vollständig zu erfassen und zu verarbeiten « und deswegen »unvollständige Bilder durch subjektive Ergänzungen vervollständigt und mit Bedeutungen versehen « (Eberle 2000, S. 91) werden.

- Dynamisch-transaktionale Ansätze versuchen die Stärken und Schwächen der medienzentristischen Ansätze und des »Uses-and-Gratifications-Ansatzes« auszubalancieren und dadurch sowohl die qualitative Dramatik der Medienprodukte selbst als auch die handlungsmotivierten Grundhaltungen der RezipientInnen empirisch zu fassen. Damit können die Interdependenzen zwischen »Medienbotschaft und aktiver Interpretation sowie zwischen Wissen und Motivation « Gegenstand empirischer Beobachtung werden und erklären helfen, »warum unterschiedliche Inhalte verschiedene Nutzer unterschiedlich ansprechen und verschieden stark aktivieren können « (Eberle 2000, S. 85 f.). Die Komplexität dieser theoretischen Perspektive erzeugt allerdings enorme methodische Operationalisierungsprobleme, die dazu führen, dass empirische Projekte sich bisher scheuen, diesen Ansatz verstärkt zu würdigen.

- Handlungstheoretische Ansätze präzisieren die Idee, dass der Medienrezeption Formen sozialen Handelns zu Grunde liegen und die RezipientInnen medialer Produktionen Medienwelten konstituieren, die ihrerseits wiederum integraler Teil moderner Lebenswelten darstellen - Jugendliche sowohl Objekt wie Subjekt des Sozialisationsprozesses und der hier zugehörigen Medien sind (vgl. Lukesch 1999). Theoretische Stützpfeiler derartiger Theorieperspektiven stellen der Symbolische Interaktionismus ebenso dar wie phänomenologische Konzepte und wissenssoziologische Überlegungen (vgl. Schröer 1994). Handlungstheoretische Theorieperspektiven gehen davon aus, dass »Medien (...) mit ihren Botschaften nur Gegenstände, Handlungen und Ereignisse anbieten, die von den handelnden Individuen zu Objekten ihrer Umwelt gemacht werden (...) Menschen nehmen mediale Botschaften wahr, interpretieren sie vor dem Hintergrund eigener Ziel- und Wertsetzungen, Pläne und Absichten und thematisieren sie, oder auch nicht. Sie sind damit 'sub- 
jektive Produzenten handlungsrelevanter Botschaften' « (Eberle 2000, S. 105). Deutlicher noch als dynamisch-transaktionale Ansätze versprechen handlungstheoretisch fundierte Modelle damit sowohl die NutzerInnenseite wie auch die medialen Produktformen umfassend - quasi multiperspektivisch - sondieren zu können (vgl. auch u. a. Schorb/Mohn/Theunert 1991). Der Nachteil dieses Ansatzes zeigt sich ebenfalls in den Grenzen seiner forschungsmethodologischen Operationalisierbarkeit. Die Rekonstruktion der subjektiven Rezeptionsformen und -inhalte, Sinnhaftigkeiten und Deutungsmuster sowie der ihr zu Grunde liegenden Handlungsmotive erfordert ein umfangreicheres methodisches Setting als dies gängigen Medienwirksamkeitsbemühungen zur Verfügung steht, insbesondere wenn darüber hinaus die Prämisse angelegt wird, dass Kinder und Jugendliche Medieninhalte auch als bezugnehmendes Muster zum Verstehen ihres gelebten Alltags heranziehen. Soll dieser Aspekt in eine handlungstheoretische Perspektive eingewoben werden, ist diese unausweichlich um eine biographietheoretische Sichtweise zu vervollständigen.

Die »Neuen« wie auch die alten Medien sind aus dem Alltag Jugendlicher nicht mehr weg zu denken. Um ihre Bedeutung für die Sozialisationsbedingungen und -kontexte sowie die subjektiven Lebensempfindungen Jugendlicher schlüssig eruieren zu können, scheinen multiperspektivische, triangulierende, ideologiekritisch fundierte handlungs- und biographiebezogene Theorie- und Forschungsmodelle monokausalen Theorieperspektiven an Erklärungskraft weit überlegen (vgl. u. a. Hopf 2001), zumal dann, wenn sie nicht isoliert von allgemeinen jugendtheoretischen Ortbestimmungen angesiedelt sind.

\section{Soziale und kulturelle Freizeitpraxen von Kindern und Jugendlichen}

»Seit vielen Jahren bemühen sich Jugendpflege und Volksbildung um die Freizeitgestaltung der Jugend. (...) Doch trotz aller Bemühungen werden bisher schätzungsweise nur 30 bis 40 von Hundert der Jugendlichen von diesen Bemühungen erfasst. Wo aber bleiben die Übrigen 60 von Hundert? Welche Bedeutung hat die Freizeit für diese nicht organisierten Jugendlichen? (...) Was treibt die große Mehrheit der Großstadtjugend in den Stunden, die ihnen die Arbeit lässt? (...) Welche Rolle kommt Kino, Theater, Radio und Musik zu? (...) Wandert sie, treibt sie Sport oder sucht sie den Rummelplatz auf? (Dinse 1932, S. 11). Übersehen wir unkommentiert den pädagogisch motivierten Impetus dieser Diagnose, dann haben sich im Kern die Fragen, die den forschenden Blicken bezüglich des jugendlichen Freizeitverhaltens zu Grunde liegen, seit der Ersten großen, auf 5.000 Aufsätzen basierenden Studie zum »Freizeitleben der Großstadtjugend « von R. Dinse (1932) bis heute nur wenig verändert. Die Frage, wie Jugendliche die Zeit gestalten, über die sie frei verfügen können, ist möglicherweise sogar der »Klassiker« der Jugendforschung schlechthin, zumindest scheint sie jene zu sein, die sich in den letzten achtzig Jahren als diejenige herauskristallisierte, die den Mainstream jugendzentrierten empirischen Interesses sowohl von quantitativen Panoramastudien (vgl. u. a. Jugendwerk der Deutsche Shell 1955, 1966, 1975, 1985/Bd. 2, 1992; Blücher 1956; Rosenmayr/Köckeis/Kreutz 1966; Silbereisen/ Vaskovics/Zinnecker 1996) als auch, wenn auch in einem bescheideneren Umfang, von qualitativen Detailstudien (vgl. u. a. Schultz 1912; Dehn 1929; Schmidt 1934; Bals 1962; Clarke u. a. 1979; Friebel 1979; Thole 1991; Bohnsack u. a. 1995; Eckert/Reis/Wetzstein 2000) bestimmt. 
Die Interdependenzen zwischen Freizeitinteressen und Freizeitbedürfnissen, Freizeithäufigkeiten, -präferenzen sowie erlangter Befriedigung wurden von der Jugendforschung schon früh aufgegriffen und erstmals von V. Blücher $(1956,1966)$ konzeptualisiert. Seine Erkenntnis, dass Freizeitinteressen von Jugendlichen nur zum Teil in dem empirisch erhobenem Freizeitverhalten wieder zu finden sind, mündete in dem Vorschlag, zwischen »harten« und »weichen « Aktivitätsformen zu unterscheiden: »Je stärker das ,Hobby', umso ,härter' die Bewusstseinsverankerung; je selbstverständlicher die Tätigkeit, umso ,weicher' die Bewusstseinsreaktion« (Blücher 1956, S. 65; auch Blücher 1966, S. 215). Welche Zuschreibung eine Aktivität schließlich erfährt, hängt von subjektiven Beweggründen, aber auch von der charakteristischen Semantik des Interessengebietes ab. Bücher lesen, sportive Praxen, Basteln und Handarbeiten sowie Musizieren können demnach als im Bewusstsein verankerte und dementsprechend oft ausgeübte, Tanzen und Kinobesuche als Interessengebiete mit einer weichen bewusstseinsmäßigen Verankerung charakterisiert werden. Unter erweiterter Perspektive ist wohl möglich zu konstatieren, dass Freizeitaktivitäten von Jugendlichen, die eine hohe Integration in den Alltag genießen, zum selbstverständlichen, unhinterfragten Handeln gehören, und somit eine geringere kognitive Erinnerungsspur hinterlassen als Freizeitformen, die nicht im Strom des Alltagstreibens verwoben sind, besondere Materialien und damit exklusiverer Aufmerksamkeit und Konzentration bedürfen (vgl. auch Strzelewicz 1965).

Die Strukturierungs- und Bündelungsversuche jugendlicher Interessen an und Aktivitäten in der Freizeit sahen sich ab der zweiten Hälfte der 1960er-Jahre mit grundlegenden Kritiken konfrontiert. Explizit wurde ihnen eine Missachtung klassen- bzw. schichtspezifischer Variabeln und Unterschiede vorgehalten (vgl. u. a. Neidthardt 1970; Lessing/Liebel 1974; Backhaus-Starost/Backhaus 1975; Onna 1976). Ein die kritischen Einwände reflektierender Versuch, jugendliche Freizeitaktivitäten strukturell zu bündeln, liegt mit der Jugendstudie der Deutschen Shell (1985) vor. In Anlehnung an zivilisations- und kulturtheoretische Konzepte wurde hier entlang der Phänomenologie des Alltags zwischen »Entspannungstechniken«, »Alltagsflips«, sportiven und kulturell-ästhetischen Freizeitpraxen von Jugendlichen unterschieden. Neuere qualitativ-empirischer Studien und zusammenfassende Beiträge zur Ausfächerung jugendkultureller Stile knüpfen hieran an, nutzen jedoch darüber hinaus auch ihr Wissen über die Alltags- und Stilorientierungen von Jugendlichen für Beschreibungen des jugendlichen Freizeitverhaltens (vgl. u. a. Strzoda1996; Ferchhoff/ Neubauer 1997).

\section{1 Über welche Ressourcen können Jugendliche verfügen? - »Zeit« und »Geld «}

Im Verlauf der letzten einhundertfünfzig Jahre hat sich für diejenigen Jugendlichen, die nicht das Privileg genossen, nach einer verlängerten Schulzeit zu studieren, die jährliche Lohnerwerbsarbeitzeit mehr als halbiert, von 3.929 im Jahr 1850 auf gut 1.600 Stunden in den 1990 Jahren. Verfügten erwerbstätige Jugendliche in den 30er-Jahren des letzten Jahrhunderts noch lediglich über 6 bis 10 Urlaubstage pro Jahr, so können sie zu Beginn des 21. Jahrhunderts auf zumeist mehr als dreimal so viele Urlaubstage verweisen. SchülerInnen können gegenwärtig über durchschnittlich 90 Schulferientage verfügen (vgl. u. a. Jaide 
1988). Generell hat sich damit objektiv die Zeit, über die Jugendliche frei verfügen können, seit der Herausbildung einer eigenen Generationsgestalt Jugend mehr als verdoppelt. Dass vor diesem Hintergrund insbesondere ältere Generationen die Jugendzeit mit »Freizeit« synonym setzen, ist allzu verständlich. Sie erinnern zugleich daran, dass »Freizeit « in der heutigen Form selbst ein Ergebnis der Moderne ist, setzt sie doch die Trennung von verpflichtenden, zumeist durch abhängige, produktionsfunktionale Erwerbsarbeit ausgefüllte oder schulisch gebundene Zeitkontingente sowie frei gestaltbare Zeiteinheiten voraus. »Solche moderne, industriegesellschaftliche Freizeit kann in einzelnen Zügen bis weit ins Kaiserreich zurückgeführt werden. Jedoch gewann sie ihre charakteristische Ausformung vor allem nach dem Ersten Weltkrieg« (Peukert 1987, S. 190).

Übereinstimmend teilen aktuellere Jugendstudien mit, dass seit den drei Jahrzehnten die frei gestaltbare Zeit für Jugendliche ein fast konstantes Niveau aufweist. Im Durchschnitt konnten und können sie werktags über vier bis acht Stunden, samstags durchschnittlich über etwas mehr als acht Stunden und sonntags über knapp 10 Stunden frei verfügen (vgl. u. a. Krüger 1991; Strzoda/Zinnecker 1996). Demzufolge haben Jugendliche an dem gesellschaftlichen Programm der strukturellen Veränderung der Zeitdispositionen (vgl. Negt 1984), also unter anderem an der Verkürzung der wöchentlichen und jährlichen Arbeitszeit, in den westlichen Bundesländern der alten Bundesrepublik in den zurückliegenden 20 Jahren nicht mehr partizipieren können (vgl. u. a. Strzoda/Zinnecker 1996, S. 284).

Für das Ende des letzten Jahrzehnts weisen Zeitbudgetstudien allerdings deutliche Unterschiede innerhalb der Jugendgeneration aus. Jugendliche in den westlichen verfügen gegenüber Jugendlichen aus den östlichen Bundesländern auch nach der »Wende « über mehr disponible Zeit, SchülerInnen erweisen sich insgesamt gegenüber Jugendlichen in Arbeitsund Ausbildungskontexten deutlich privilegiert, SchülerInnen in den östlichen Bundesländern verwenden mehr Zeit täglich für die Schule als ihre Altersgleichen in den westlichen Bundesländern und weibliche können gegenüber männlichen Jugendlichen deutlich geringere Zeiten frei gestalten. Vorsichtig notieren aktuellere Zeitbudgetstudien auch einen Wandel der StudentInnenrolle, signalisieren, dass das »Studium nicht mehr allein im Vordergrund der Zeitverwendung steht, sondern mit anderen Tätigkeiten« (vgl. Strzoda/Zinnecker 1996, S. 299) inzwischen in gewisser Art und Weise zu »konkurrieren« scheint.

Die ökonomischen und materiellen Ressourcen, die der Jugendgeneration insgesamt zur Verfügung stehen und ihr die Teilnahme am vergesellschafteten Freizeit- und Konsummarkt ermöglichen, haben sich von den 70er bis zu den 90er-Jahren des letzten Jahrhunderts nahezu verdoppelt, von 17 Milliarden DM 1977 auf zirka 31 Milliarden DM 1989 (Bravo-Studie 1986; G+J 1986; vgl. zusammenfassend Wolsing 1991), und im Vergleich zu den 60er-Jahren (vgl. Scharmann 1965, S. 23) nahezu verzweieinhalbfacht. 1999 warb die Europäische Jugendmesse GmbH für die von ihr veranstaltete Jugendmesse »YOU « zukünftige Aussteller mit dem Hinweis, dass in »keiner Altersgruppe Marken so schnell und mit so geringem Aufwand 'zu machen' sind wie im Jugendbereich. Über knapp 50 Milliarden DM jährlich verfügt die Jugend in Deutschland selbstständig und noch mehr Geld bewegt ihr Einfluss auf Kaufentscheidungen innerhalb der Familien« (You-Team- 
Messe 2001). Konnten SchülerInnen in den 1950er-Jahren über durchschnittlich etwas mehr als 20 DM (Blücher 1956, S. 47) und in den 1960er-Jahren über DM 35 DM (vgl. Blücher 1966, S. 16) im Monat frei entscheiden, so können sie Anfang der 90er-Jahre des letzten Jahrhunderts durchschnittlich Beträge zwischen 90 DM (Wolsing 1991, S. 176) und 115 DM (Behnken u. a. 1991, S. 134) eigenverantwortlich ausgeben (zur Entwicklung in der früheren DDR vgl. Mitksch 1972, S. 46 f.). Insbesondere das eigenverfügbare Geldvolumen von älteren Jugendlichen vervielfachte sich in den letzten Jahrzehnten im Durchschnitt, obwohl seit gut 20 Jahren immer weniger ältere männliche wie weibliche Jugendliche über ein eigenerwirtschaftetes Einkommen frei bestimmen können. Die zu den materiellen Ressourcenlagen vorliegenden Durchschnittswerte verdecken allerdings soziale, geschlechts-, alters- und milieuspezifische Unterschiede sowie die Tatsache, dass nichtdeutsche Kinder und Jugendliche und die Heranwachsenden in den östlichen Bundesländern immer noch über deutlich geringere finanzielle Möglichkeiten verfügen als ihre AltersgenossInnen durchschnittlich in den westlichen Bundesländern (vgl. Jugendwerk der Deutschen Shell 2000, S. 286).

Deutlich zugenommen hat aufgrund der durchschnittlichen materiellen Verbesserungen in den letzten Jahrzehnten die Medienausstattung und die Ausstattung mit anderen konsumtiven Gütern (vgl. Behnken u. a. 1991; Wolsing 1991; Fritzsche 2000). Zu Beginn des 21. Jahrhunderts ist davon auszugehen, dass nur noch eine verschwindend kleine Minderheit der Jugendlichen und auch zunehmend weniger Kinder von der Möglichkeit, die klassischen Medien - Radio, Schallplatten und Kassettenrekorder, Fernseher und Fotoapparat täglich zu nutzen, ausgeschlossen ist. Auch im Bereich der »Neuen Medien« (Video, CDPlayer und Heimcomputer) ist seit 20 Jahren ein Zuwachs im persönlichen Besitz dieser Medien bei Jugendlichen zu verzeichnen. Inzwischen besitzen $30 \%$ aller deutschen Heranwachsenden einen Computer, den sie alleine benutzen können, und weitere $28 \%$ können zumindest über einen solchen mit verfügen (vgl. Fritzsche 2000, S. 200). Über $97 \%$ aller Jugendlichen sowohl in den östlichen als auch in den westlichen Bundesländern besitzen ein Radio sowie ein Fernsehgerät im bewohnten Haushalt, über $66 \%$ in den westlichen und über $41 \%$ in den östlichen eine Hifi-Anlage, über $42 \%$ in den westlichen und über $47 \%$ in den östlichen einen Radiorekorder, über $70 \%$ in den westlichen und $41 \%$ in den östlichen einen Plattenspieler, gut $45 \%$ in den westlichen und etwas weniger als $32 \%$ in den östlichen einen Videorekorder und über $30 \%$ in den westlichen und knapp $14 \%$ in den östlichen einen CD-Player. Inzwischen können auch $28 \%$ aller deutschen, $41 \%$ aller italienischen und $28 \%$ aller türkischen 15- bis 24-jährigen Jugendlichen auf ein eigenes Handy zurückgreifen. Wenig überraschend, dennoch auffällig, dass weibliche Jugendliche beim Besitz »Neuer Medien«, insbesondere aber beim Besitz und somit auch bei der Benutzung von Computern, weitestgehend ausgegrenzt bleiben (Baacke u. a. 1990; Eckert u. a. 1991, S. 64; Fritzsche 2000).

Generell gilt, dass jüngere Jugendliche über geringere finanzielle Ressourcen verfügen als ältere, die eigenverfügbare Geldmasse von Mädchen geringer ist als die von Jungen, die materielle Kapitaldisposition der Eltern einerseits, andererseits der Zeitpunkt des Übergangs in das Berufsleben unterschiedliche materielle Ressourcenlagen bedingen. Die mate- 
riellen, finanziellen, häuslich-räumlichen, mobilen und zeitlichen Ressourcen, die Jugendlichen heute zur Verfügung stehen, scheinen allerdings nicht mehr wie noch bis in die 70er-Jahre hinein ausschließlich und durchgehend schichtenspezifisch vermittelt zu sein. Diese, vornehmlich auf die altbundesrepublikanischen Länder zutreffende Feststellung stützt sich auf sozialwissenschaftliche Diagnosen, die von einer Diversifizierung der Lebenslagen und einer Ausdünnung sozialer Klassen und Schichten bei gleichzeitiger, sukzessiver »Verschärfung sozialer Ungleichheiten « (Beck 1986) ausgehen. Soziale Ungleichheiten formulieren sich im Jugendalter nicht mehr ausschließlich über das »Haben«, sondern die sozialen und intellektuellen Kompetenzen, die über Art und qualifizierten Umgang mit den Ressourcen befinden, gestalten auf einem quasi kulturell vermitteltem Niveau soziale Ungleichheiten heute mit. Dieser generelle Trend scheint ungehindert der Tatsache, dass sich in der zweiten Hälfte der letzten Dekade soziale Ungleichheitslagen deutlich verschärften und Kinder und Jugendliche aus den fünf östlichen Bundesländern sowie aus strukturschwächeren Regionen, Jugendliche ausländischer Nationalität und weibliche Jugendliche, trotz Ausdehnung ihrer Optionen, vornehmlich auf der Verliererseite zu finden sind, zu Beginn des neuen Jahrtausend zu konsolidieren.

\subsection{Zwischen aktiver Selbstverwirklichung und entspannenden Konsumpraxen - Freizeitorientierungen von Jugendlichen}

Jugendliche Lebenswelten kennzeichnet zu Beginn des neuen Jahrtausends eine expandierende Vervielfältigung und Ausdifferenzierung kreativer und konsumtiver Freizeittätigkeiten. Die Pluralisierung und Ausfächerung kultureller Praxen von Jugendlichen potenzierten die schon bestehende Unüberschaubarkeit des Freizeitangebots nochmals. V. Blüchers (1967, S. 220) Markierung, dass »das Freizeitsystem (...) vielgestaltig, schwer zu übersehen und auf einen Nenner zu bringen « ist, hat an Aktualität keine Einbuße erfahren - im Gegenteil: Viele ehemals »exotische« Freizeitpraxen wurden zu einem genuinen, integrierten, ja fast unauffälligen Bestandteil des Alltags - verloren quasi ihre »Unschuld «.

- Seine »Unschuld« verloren hat sicherlich auch der Konsum von Alltagsdrogen. Aus Sicht des Abstinenzgedanken ist hier für die letzte Dekade eine erfreuliche Entwicklung zu vermelden. Der Anteil der 18- bis 25-jährigen RaucherInnen an der altersgleichen Bevölkerung sank in den letzten gut 25 Jahren von $62 \%$ auf $49 \%$ und der der 12- bis 17-jährigen von $36 \%$ zu Beginn der 1970er-Jahre auf knapp $26 \%$ am Ende der 1990er-Jahre. Und tranken in den 70er-Jahren von den 12- bis 25-jährigen $42 \%$ mindestens einmal die Woche Bier, so waren es knapp 30 Jahre später lediglich noch $27 \%$ (Kolip 2000). Eine diesem Trend entgegen laufende Entwicklung wird jedoch für die fünf östlichen Bundesländer notiert. Hier legen neuere Befunde nahe, davon auszugehen, dass im Nachtransformationsprozess der Genuss sowohl von legalen wie von illegalen Genussmitteln und Drogen - auch bedingt durch die neuen Designerdrogen - stark zugenommen hat (Müller 2000). Mit Ausnahme des Tabakkonsums scheint der Gebrauch von legalen und illegalen Drogen bei Jugendlichen dennoch in den östlichen Bundesländern weniger ausgeprägt zu sein als in den westlichen (vgl. Nolternsting 1998). Trotz partieller gesellschaftlicher Skandalisierung wird das jugendliche Freizeitverhalten nicht primär von einem durchgehend expansiven Dogenkonsum getragen.

- Dem widerspricht auch nicht die Beobachtung, dass im Allgemeinen das gesellige und kommunikative Freizeitmoment im letzten Jahrzehnt deutlich an Priorität gewann und zuweilen sogar von einem »Kult der Geselligkeit«, der sich seit den fünfziger Jahren des letzten Jahrhunderts 
sukzessive etabliert hat (Strzoda 1996, S. 263), gesprochen wird. So sind - neben dem stark präferiertem »Medienkonsum « - Aktivitäten wie mit FreundInnen die Zeit verbringen, mit ihnen zu telefonieren und mit der Familie zusammen sein immer noch hoch gehandelte Aktivitäten. Jeweils über $40 \%$ bis 50\% der Jugendlichen zählen Sport treiben, Lesen sowie Spielen und Arbeiten am Computer zu ihren liebsten Freizeitbeschäftigungen, wobei das Lesen in den letzten Jahren einen leichten Rückgang erfahren - insbesondere die regelmäßige Lektüre einer Abonnementszeitung ist in den letzten zwanzig Jahren des verstrichenen Jahrhunderts deutlich zurückgegangen, von knapp $64 \%$ regelmäßigen LeserInnen auf gut $44 \%$ (Noelle-Neumann 1999, S. 24) und die Beschäftigung mit dem Computer als Freizeitaktivität an Bedeutung gewonnen hat. Und auch die Gartenarbeit erhält inzwischen ebenfalls eine geringere Präferenz. In Bezug auf die musikalische Orientierung fällt auf, dass die Gitarre die Blockflöte als das am häufigsten gespielte Musikinstrument abgelöst hat. Insgesamt zeigt sich eine Höherbewertung der Musikinstrumente durch Jugendliche, die auch von den musikalischen Idolen der Jugendlichen vorgeführt werden. Die hier schon offenkundige Tendenz dokumentiert sich noch deutlicher bei den Tänzen. Neben der Reanimierung klassischer Tänze ist die Herausbildung einer neuen, um sportive und kulturell-ästhetische Facetten angereicherten Tanzkultur mit starken Event-Charakter zu erkennen »Disco is death « (Geene 2001), erlebt aber in kleinen Bars ebenso wie in alten Lager- und Fabrikhallen täglich eine Wiedergeburt.

- In der ersten Shell Jugendstudie des soeben angebrochenen neuen Jahrhunderts werden diese allgemeinen Daten präzisiert: $98 \%$ aller 14- bis 24-jährigen Jugendlichen gehen mehr oder weniger regelmäßig auf Partys oder Feste und immerhin noch $68 \%$ arbeiten oder spielen regelmäßig am Computer. Diese Präferenzen korrelieren mit dem Befund, dass 47,7\% der männlichen und $60,9 \%$ der weiblichen Jugendlichen im Westen und $60 \%$ der männlichen und 71,8\% der weiblichen Jugendlichen in den östlichen Bundesländern gern beziehungsweise sehr gern eine Diskothek besuchen (vgl. Nolteernsting 1998). Feiern und »Party machen« stellen Aktivitäten dar, die nach Angaben der älteren Kinder und Jugendlichen zu $42 \%$ gemeinsam mit nicht-deutschen Heranwachsenden ausgeübt werden (vgl. Jugendwerk der Deutschen Shell 2000, S. 207). Immerhin noch 19,7 \% der männlichen und 15,8 \% der weiblichen Jugendlichen im Westen und 22,9\% der männlichen und 20,8 \% der weiblichen Jugendlichen in den östlichen Bundesländern statten gern beziehungsweise sehr gern Jugendzentren einen Besuch ab - in der Altersgruppe der 15- bis 17jährigen votieren dahingehend sogar jeweils über $30 \%$ der befragten Jugendlichen (vgl. Nolteernsting 1998).

- Insgesamt kann Unsicherheit darüber bestehen, ob die heutige Jugendgeneration unpolitischer ist als ihre Vorgängergenerationen oder ihre politischen Artikulationsbedürfnisse nicht nur anders, intuitiver, projekt- und situationsbezogener kundtut. Einigkeit hingegen dürfte darin schnell hergestellt sein, dass sie sich im Kern spaß- und lebensfroher artikuliert und stets zu wissen scheint, wo die Nächste »paradiesische Party « stattfindet. Und auch ihre Reise- und Urlaubsfreudigkeit scheint zugenommen zu haben. Natürlich gehört die Generation der Jüngeren nicht erst seit der Zeit der »fahrenden Schüler « und dem Wanderbrauch der Handwerksgesellen zu der reise- und urlaubsfreudigsten Bevölkerungsgruppe. Doch die Reiseintensität der 14- bis 24-jährigen war wohl noch in keiner Epoche so ausgeprägt wie in der jetzigen. Der Grund hierfür ist allerdings weniger in einem Mentalitätswandel, sondern wahrscheinlich eher in der Zunahme der finanziellen Ressourcenlagen zu vermuten. Über 60 \% der 14- bis 17-jährigen Jugendlichen haben Mitte der 90er-Jahre eine Urlaubsreise von einer mindestens fünftägigen Dauer realisiert (vgl. Opaschowski 1996).

- $96 \%$ aller Jugendlichen hören regelmäßig Musik (vgl. Jugendwerk der Deutschen Shell 2000, S. 207). In den 90er-Jahren des letzten Jahrhunderts schienen jedoch gerade die jugendlichen Musikkulturen in eine unangenehme »Zwickmühle« geraten zu sein. Auf der einen Seite gibt es die Zwänge der ständig expandierenden Musikindustrie, auf der anderen Seite die »neuen Rebellen«, die mit ihrem »Nazi-Rock« das subversive und aggressive Element der Rockmusik immer 
mehr für sich zu vereinnahmen trachten und über Skinbands wie »Störkraft«, »Radikahl« und »Kraftschlag« mit ihrem »ultraharten Sound « partiell inzwischen das verkörpern, was Rock'n Roll schon immer sein wollte. Zeigten jedoch bis zu Beginn der 90er-Jahre des letzten Jahrhunderts vornehmlich - wenn auch nicht ausschließlich - männliche Jugendliche über 15 Jahre eine besonders hohe Affinität zu Rockgruppen und waren insbesondere sie es, die die jugendliche Musikkultur zu einer Jugendkultur erweiterten, so sind es heute auch und insbesondere jüngere Mädchen, die über die »Boygroups« angeregt, eigenständige jugendkulturelle Stile kreieren: »In Deutschland der 90er-Jahre grassiert eine neue kollektive Kinderverzückung. Zwar zählen kreischende Mädchen, Ohnmachtgefühle und Massenhysterie seit den wilden Zeiten der Beatles in den 60er-Jahren zu den gewöhnlichen Spaßritualen der Jugendkultur - noch nie aber hat sich ein ganzes Pop-Genre derart erfolgreich der mutwilligen Erregung von Teenagerkrawallen (...) verschrieben « (Der Spiegel 1997, S. 186). Verkörperten in den 1990er-Jahren Bands wie »New Kids on the Block «, »Caught In The Act« und die »Backstreets Boys« das aktuelle Zeitgefühl, so sind es zu Beginn des neuen Jahrhunderts Gruppen wie »Toploader«, »No Angels« und »Outkast«, die in der Beliebheitsskale hoch rangieren und insbesondere jüngere Mädchen begeistern, auch weil sie in einem hohen Maße Identifikationswünsche freisetzen und adoleszente Fantasien bedienen. Musik, Pop und Rock sind heute allerdings keineswegs mehr Synonyme für das jugendliche Emanzipationspotenzial, für Nonkonformismus und Fortschritt. Die Geschichte »des jungen Mannes, der zur Gitarre griff, eine Band gründet und Musik spielt, die seine Eltern und die Gemeinschaft der Erwachsenen provoziert« (vgl. Holert/Terkissidis 1996, S. 5), ist inzwischen Mythos.

- Sport gehört auch im neuen Jahrhundert zu den attraktivsten jugendlichen Freizeitbeschäftigungen $-81 \%$ der befragten Jugendlichen geben an, Sport zu treiben (vgl. Jugendwerk der Deutschen Shell 2000, S. 207). Vieles spricht dafür, in dem jugendlichen »Sport treiben« und den gewählten Optionen für bestimmte Sportarten eine der gravierendsten Veränderungen der letzten anderthalb Dekaden zu sehen. Über $23 \%$ der Jugendlichen geben an, fast jeden Tag, $27 \%$ zweibis dreimal die Woche und $19 \%$ zumindest einmal in der Woche außerhalb eines Vereins sich sportlich zu betätigen - damit treiben immerhin knapp $70 \%$ der Jugendlichen mindestens einmal die Woche unabhängig von einem Verein Sport. Lediglich $38 \%$ der befragten Jugendlichen aktivieren sich innerhalb eines Sportvereins. Die sportorientierten Präferenzen von Jugendlichen haben sich jedoch nicht nur enorm vervielfacht, sondern auch deutlich von ihrer bisherigen Fokussierung auf den Schul- und Vereinssport gelöst. Einige der spielerischen Bewegungsaktivitäten wie beispielsweise Fahrrad fahren, Mountainbiking, Skaten, Kickboard fahren, Wandern und Yoga werden von vielen nicht einmal mehr dem Sport zugeordnet (vgl. Brinkhoff 1998), obwohl sie wie das Fahrrad fahren vor dem Schwimmen, Fußball und Jogging die Ranking Liste der ausgeübten sportlichen Aktivitäten außerhalb der Schul- und Vereinskultur anführen. Insbesondere neue Trendsportarten erfreuen sich einer zunehmenden Akzeptanz. Bei den vereinsgebundenen Betätigungen rangiert bei den männlichen Jugendlichen zwar immer noch der Fußballsport mit Abstand an der ersten Stelle, jedoch haben Tennis, Handball und Basketball deutlich an Zuspruch gewonnen. Ausgeprägter ist der Diversifizierungsprozess allerdings bei den weiblichen Jugendlichen dokumentiert, wo der Pferdesport nur noch knapp vor Tennis, Schwimmen und Volleyball die Liste der ausgeübten Sportarten anführt. Auch bezüglich des Sport treibens zeigen sich damit deutliche geschlechts- und bildungsaspirationsabhänge Verteilungen: Jungen betreiben immer noch wesentlich häufiger Sport als Mädchen und ebenfalls sportaktiver sind GymnasiastInnen als Real- und HauptschülerInnen (vgl. Brinkhoff 1998).

Erstmals Mitte der 1980er-Jahre wurden jugendliche Freizeitformen entlang ihrer alltagsphänomenologischen Ausfächerung unter den Etiketten »Ausruhen/Erholung « einerseits und den »Flip-Praxen« andererseits sortiert (vgl. Fuchs 1985, S. 7 ff.). Offensichtlich ist, dass im »Supermarkt« der sich ständig wandelnden und kommerzialisierenden Freizeitan- 
gebote Jugendliche - zunehmend jedoch auch ältere Kinder - expressive »Flip-Praxen« entfalten. Es sind dies für die Heranwachsenden »kleine Fluchten« aus der Alltagsroutine. »Musik irrsinnig laut hören«, »beim Essen mal richtig sündigen«, »seinen Körper mal bis zum Letzten verausgaben «, »sich in seine Liebhabereien vergraben « und »mit anderen die Nacht bis zum Morgen durchmachen« sind jugendliche Alltagsflips, die von durchschnittlich knapp der Hälfte der Jugendlichen favorisiert werden. Schule »Blaumachen « und »verrückte Sachen anziehen « nennen hingegen nur ein Zehntel der Jugendlichen. Heraus zu stellen ist, dass eine hohe Flip-Intensität aussagekräftig mit dem Geschlecht, auch mit dem Alter, jedoch weniger mit dem Schulniveau korreliert. Generell votieren Jungen für expressivere Aktionen als Mädchen. Zudem zeigt sich: Jugendliche mit einer hohen FlipIntensität scheinen geselliger zu sein - Geselligkeit wird von Mädchen durchgängig mindestens so stark präferiert -, bevorzugen außerhäusliche Aktivitäten und sind sportlich engagiert, in ihrer Freizeit extrovertierter und in ihren Freizeitpräferenzen vielfältiger (vgl. Fuchs 1985, S. 28 ff.; vgl. auch Jugendwerk der Deutschen Shell 2000).

Zunehmend schwieriger ist die Verortung bestimmter Freizeitpraxen und kultureller Stilmuster nach altersspezifischen Präferenzen. Die frühe Zwangs- und Wahlbiographisierung kindlicher und noch deutlicher jugendlicher Lebensläufe und die Öffnung des Freizeitmarkts für jüngere Altersgruppen haben zur Auflösung eindeutiger Altersgrenzen bei Kindern und Jugendlichen geführt und entsprechend zu einer Verflüssigung altersspezifischer Freizeitpräferenzen. Keineswegs kann jedoch von einem ausschließlich hedonistisch ausgeprägten jugendlichen Freizeitverhalten gesprochen werden, da berufliche Weiterbildung, kulturelle Interessen und Zeitung lesen mit zunehmenden Alter ebenfalls häufige Freizeithandlungen sind (Fritzsche 1997, S. 345). Das Freizeitverhalten ist neben seiner deutlich altersspezifischen Prägung gegenwärtig immer noch durch geschlechtsspezifische Unterschiede polarisiert, auch wenn diese zunehmend undeutlicher werden (vgl. u. a. Strzoda 1996, S. 261).

Die größten geschlechtsspezifischen Unterschiede zeigen sich in der Altersgruppe der 13- bis 16-jährigen. Mädchen bevorzugen stärker künstlerisch-musikalisch-literarische Aktivitäten wie Briefe sowie Tagebuch schreiben, Musik machen, Lesen und Malen. Insbesondere in der frühen Jugendphase stehen bei Mädchen Aktivitäten mit einem introvertierten und problemverarbeitenden Charakter analog zu dem Durchleben der Pubertät im Vordergrund. Weitere »typisch-weibliche « Präferenzen sind persönliche Probleme »bequatschen «, einen »Schaufensterbummel « machen - u. a. ein Indiz für die modische Orientierung vieler Jugendlicher -, und Telefonieren. Bei den 17- bis 20-jährigen Mädchen gewinnt dann der gesprächsorientierte und gesellige Aspekt an Priorität. Eher »männliche« Freizeitbeschäftigungen sind Fernsehen, Videos schauen, Computerspiele, Comics lesen und im Allgemeinen technik- und motorisierungsorientierte Freizeithandlungen. Die Freizeitpräferenzen im Verlauf der Jugendphase beider Geschlechter liegen bei den Jüngeren eher im häuslichen, während der »Hochphase« im geselligen und kommunikativen und in der Nachjugendzeit wieder vermehrt im häuslichen Bereich - deutlich ist somit der Zusammenhang zwischen ausgeübter Freizeittätigkeit und biografischer Lebenslaufetappe ausgewiesen. Mit zunehmendem Alter synchronisieren sich, auch gerade vor diesem Hin- 
tergrund, die Lebensläufe von Jungen und Mädchen. Deshalb lässt sich hier eine Annäherung zwischen weiblicher und männlicher Freizeitgestaltung beobachten (vgl. Strzoda 1996; Fritzsche 1997).

Wie haben sich die Freizeitgewohnheiten der Jugendlichen in den östlichen Bundesländern entwickelt? Verweisen die empirischen Daten auf eine Angleichung oder entwickelten sich die jugendlichen Freizeitoptionen in den westlichen und östlichen Bundesländern scherenartig weiter auseinander? Während noch 1991 vielfältige Differenzen zwischen den Freizeitmustern der west- und ostdeutschen Jugend zu erkennen waren (Jugendwerk der Deutschen Shell 1992, Bd. 2, S. 242 ff.), ist heute eine weitgehende Angleichung dokumentiert. Die Gemeinsamkeiten dokumentieren sich auffällig in den ressourcenunabhängigen Dimensionen, also in den besonders interessen-, motivations- und kompetenzabhängigen Dimensionen und in der Geselligkeitsorientierung. Die kaum noch nennenswerten Unterschiede manifestieren sich, neben den infrastrukturellen, dort, wo die verschiedenen Lebensläufe sichtbar werden, beispielsweise in dem Freizeitverhalten der jungen ostdeutschen Frauen (vgl. Fritzsche 1997; Strzoda 1996; Jugendwerk der Deutschen Shell 2000). Doch die mittels Befragungen geronnenen Befunde sind noch vorsichtig zu betrachten, ist doch nicht auszuschließen, dass sich die Differenzen, auch dort, wo auf der quantitativen Ebene eine Angleichung signalisiert wird, quasi hinter den Kulissen der großen Zahlen fortsetzen und möglicherweise bisher noch nicht entdeckte Unterschiede in den qualitativen Motivlagen fortbestehen.

\subsection{Vom tragbaren Telefunken-Radio zum eigenen Laptop - Medienverhalten und Technikorientierung von Jugendlichen}

$\mathrm{Ob}$ und wenn ja inwieweit jugendliche Freizeitaktivitäten heute von Medien beeinflusst und über sie vorstrukturiert werden, visuelle und auditive Kommunikationsformen verbale Artikulation zurückdrängen und die Lesekultur durch eine Techno- und Computerkultur abgelöst wird, ist in der Jugendforschung seit zehn Jahren sehr umstritten. Insbesondere die Tatsache, dass die traditionell von weiblichen Jugendlichen höher präferierte Freizeitform »Lesen« - als konventionelle, den bürgerlichen Bildungsidealen am nächsten stehende Freizeittätigkeit - seit den 50er-Jahren des letzten Jahrhunderts nur leicht und keinesfalls gravierend an Bedeutung verloren hat (vgl. Zinnecker 1987; Fritzsche 1997; Nolteernsting 1998; vgl. auch Abschnitt 2.2), provoziert das in diesem Bereich häufig doch sehr schablonierte Nachdenken und relativiert jene Diagnosen, die von einer durchgehenden und tief greifenden Mediatisierung jugendlicher und kindlicher Freizeitwelten ausgehen. Demgegenüber sind folgende Befunde zu konstatieren:

- Der mehr oder weniger regelmäßige Fernsehkonsum von Kindern ab dem dritten Lebensjahr scheint sich in den letzten zwei Dekaden trotz anders lautender Annahmen kaum verändert und bei einer durchschnittlich ca. 1,5-stündigen Sehdauer pro Tag eingependelt zu haben. Leicht zugenommen hat möglicherweise jedoch die Sehdauer der 6- bis 13-jährigen (vgl. Kofler/Graf 1995). Zwei Drittel dieser Altersgruppe sitzen täglich vor dem Bildschirm. Während die 12- bis 15-jährigen am häufigsten das TV nutzen, geht der Fernsehkonsum in der Altersgruppe der 16bis 19-jährigen Jugendlichen wieder deutlich zurück (Fritzsche 2000). Doch die empirischen Daten sind uneindeutig. Wird einerseits konstatiert, dass der tägliche Bilderkonsum via Fernsehen in 
der Altersgruppe der 14- bis 19-jährigen in den 1990er-Jahren deutlich abgenommen hat und inzwischen über eine halbe Stunde unterhalb der durchschnittlichen Sehdauer der jüngeren Jugendlichen (vgl. Kofler/Graf 1995) liegt, halten andere Studien fest, dass die durchschnittliche Sehdauer in den vergangenen fünfzehn Jahren um cirka $10 \%$ zugenommen hat und bei den 10- bis 13-jährigen aktuell bei cirka zwei und bei den über 14-jährigen Jugendlichen bei über drei Stunden liegt (vgl. Groebel 1999). Männliche Jugendliche sehen anscheinend gut doppelt so lange täglich »fern « wie Mädchen, u. a. möglicherweise auch dadurch bedingt, dass sie Sportsendungen in einem wesentlich ausgeprägterem Umfang konsumieren als weibliche Jugendliche. Medienstudien legen nahe, dass Kinder und Jugendliche mit einer geringeren Bildungsaspiration deutlich länger »fern «sehen und Jugendliche aus niedrigen sozialökonomischen Lebenswelten vorrangig harte Actionfilme und medial inszenierte Gewaltdarstellungen bevorzugen. Insgesamt meinten knapp $80 \%$ der 12- bis 24-jährigen, häufig oder sehr häufig Fernsehen zu schauen (vgl. Jugendwerk der Deutschen Shell 1997, 2000; Groebel 1999). Aber auch Vielseher scheinen an dieser Form der konsumtiven Freizeitbeschäftigung kein besonderes Vergnügen zu finden, sondern auf Grund mangelnder Handlungsalternativen auf sie zurück zu greifen (vgl. u. a. Eberle 2000, S. 319).

- Vorurteile und unausgewiesene Bilder bestimmen die öffentlichen Meinungen über jugendliche FersehseherInnen immer noch entscheidend mit. Die seit einigen Jahren ausgestrahlten täglichen Talkshows sowie die Reality-Formate wie beispielsweise »Big Brother« und »Girls-Camp« erfreuen sich, so die publikumswirksame Botschaft, insbesondere bei Jugendlichen einer erfreulichen Aufmerksamkeit. Den noch wenig validen Daten zufolge findet dieser Alltagsbefund in genereller Form jedoch keine empirische Sättigung. Nur wenige Sendungen - wie beispielsweise »Arabella« und »Andreas Türk - erreichen eine über 25-prozentige Beachtung bei den 12- bis 17-jährigen Jugendlichen von zumindest einmal pro Woche. $\mathrm{Zu}$ den VielseherInnen dieser Sendeformate zählen dabei eindeutig weibliche Jugendliche. Die zwischen authentischer Selbstrepräsentation und Inszenierung angesiedelten Sendungen stehen den vorliegenden Studien zufolge allerdings nicht im Zentrum der jugendlichen Fernsehnutzung und die diagnostizierbare Rezeptionshaltung schwankt zwischen »Fans«, »Orientierungssuchenden« und »KritikerInnen« (vgl. Pauls-Haase u. a. 1999, S. 375), auch weil die Ansprïche der konsumierenden Fernseh»talkerInnen « uneinhaltlich zwischen der Suche nach Amüsement, Entspannung und naiv-idealistischer Selbstverortungssuche pendelt (Pauls-Haase u. a. 1999, S. 408 ff.).

- Festzustellen ist allerdings ein kontinuierlicher Anstieg des Videokonsums bei Kindern und Jugendlichen mit zunehmendem Alter, obwohl unter den 6- bis 10-jährigen auch noch ein Drittel Nie-Seher zu finden ist und unter den 14- bis 16-jährigen immerhin noch knapp ein Viertel keine Videofilme sieht. Einschränkend zu konstatieren ist jedoch, dass die Häufigkeit des Konsums von audiovisuellen Produktionen keine Aussage über die Qualität und die Tiefe der Rezeption erlaubt. In der Bundesrepublik Deutschland sahen Mitte der 1990er-Jahre 9- bis 17-jährige täglich cirka 16 Minuten Video, hörten knapp eine Stunde Radio, hörten cirka 50 Minuten Tonkassetten oder CDs und verbrachten durchschnittlich acht Minuten nicht spielend und cirka 20 Minuten spielend vor dem PC (vgl. Krotz 1999, S. 159)

- Trotz des steigenden Einflusses von Bildmedien muss nicht unbedingt von einer Visualisierung des kindlichen und jugendlichen Freizeitverhaltens gesprochen werden, zumal auditive Medien mit zunehmendem Alter für Kinder und Jugendliche immer attraktiver werden. Insbesondere Musiksendungen im Radio erfreuen sich großer Beliebtheit, und etwa ab dem 16. Lebensjahr wird ihnen mehr Zeit gewidmet als dem Fernsehen. Für die aktuelle und zukünftige Printmedienforschung in Bezug auf Kinder und Jugendliche dürften vor allem nach Alterskohorten, bildungsspezifischen sowie geschlechtsspezifischen Aspekten differenzierende Analysen von Belang sein.

- Die »Neuen Medien« sind in der letzten Dekade des letzten Jahrhunderts zum selbstverständlichen Bestandteil des jugendlichen Alltags geworden. Medienpädagogische Untersuchungen deu- 
ten z. B. darauf hin, dass Jugendliche zur Computerwelt ein sehr pragmatisches Verhältnis entwickelt haben. Mehr als ein technologischer Sprung ist der »Siegeszug « der Handcomputer, dessen wohl immer noch bekanntester Typ »Game Boy « zwar vermehrt auch von Mädchen zum Spielen genutzt wird, vorrangig aber das Spielzeug von männlichen Jugendlichen ist. Der schon für das Videospiel seit Mitte der 1980er-Jahre konstatierte hohe Faszinationsgehalt mit einer enormen Abschirmungsfunktion gegenüber dem »grauen Alltag « erreichte erstmals durch die rasante Verbreitung von Handcomputern wie dem »Game Boy« eine neue Dimension. Inzwischen haben »Spielkonsolen« den Markt neu gemischt und einfache Spiele in Handys und Taschenrechner erfreuen sich als Freizeitbeschäftigungen »für zwischendurch« einer großen Beliebheit. Inwieweit diese »technologischen Sprünge « Freizeitverhalten und -orientierungen von Kindern und Jugendlichen grundlegend beeinflussen und neu strukturieren, ist nicht zu prognostizieren und bisher noch wenig erforscht. Zumindest deutlich angezeigt ist, dass Jugendliche, die am Computer programmieren, weniger Videoproduktionen sehen als die Jugendlichen, die dem nie nachgehen. Im Vergleich zu den jugendlichen Nicht-Programmierern besitzen prozentual die jugendlichen Häufig-Programmierer durchschnittlich 70 Bücher mehr. Jugendliche, die mit dem Computer umgehen, sind entgegen landläufiger Meinungen auch nicht weniger kreativ. Jungen, die mit diesem Medium umgehen, Malen häufiger als Nicht-Computerbenutzer, und »Computer«-Mädchen Malen, Basteln, schreiben Tagebuch und Briefe, verfassen Gedichte und Musizieren häufiger als ihre nicht »computerisierten « Altersgenossinnen (vgl. Baacke u. a. 1990, S. 239 ff.). Insgesamt können allerdings lediglich $7 \%$ aller weiblichen, jedoch $19 \%$ aller männlichen Heranwachsenden der Altersgruppe der 9- bis 17-jährigen auf einen direkten Zugriff auf einen Computer in ihrem eigenen Zimmer verweisen. Insgesamt können $14 \%$ aller 9- bis 17-jährigen Heranwachsenden über einen PC mit CD-ROM Laufwerk im eigenen Zimmer und weitere $39 \%$ über einen solchen in dem von ihnen mitbewohnten Haushalt verfügen (Krotz 1999, S. 160).

- Der Computer mit seinen weltumspannenden Interaktionsmöglichkeiten hat zu Beginn des 21. Jahrhunderts das Fernsehen als technisches Leitmedium abgelöst. Diese Befunde korrelieren mit neueren Abfragen, wonach $60 \%$ aller Hauptschüler und $25 \%$ aller HauptschülerInnen, $74 \%$ der männlichen und $59 \%$ der weiblichen BesucherInnen von Realschulen und $77 \%$ der männlichen und $72 \%$ der weiblichen GymnasistInnen auf einen Internetzugang zurückgreifen können und diesen auch pro Woche zwischen 4 Stunden - männliche Hauptschüler - und 14 Stunden männliche Gymnasiasten - nutzen (vgl. Harberg 2001). Aktivitäten wie am Computer spielen, Texte schreiben und für die Schule arbeiten gehören neben Malen und Grafiken erstellen zu den am Meisten ausgeübten Tätigkeiten am Computer. Sicherlich gibt es auch die isolierten, in sich gekehrten, fast schon »pathologischen « VielnutzerInnen eines Mediums, jedoch treten entsprechende Extremfälle in quantitativen Untersuchungen kaum in Erscheinung (vgl. Baacke u. a. 1990, S. 248; vgl. auch Jugendwerk der Deutschen Shell 2000).

Bezüglich der sozialisatorischen Bedeutung aggressiver medialer Produktion wird in den letzten Jahren verstärkt darauf hingewiesen, dass »es zweifellos einen bestimmten Anteil von Heranwachsenden - nach meinen Untersuchungen liegt dieser bei etwa $20 \%$ - gibt, die durch medial inszenierte Gewalt in ihrem emotionalen und sozialen Entwicklungsprozess behindert werden. Aber: Diese Gefährdung wird nicht durch die Medien erzeugt, sondern durch die Mediennutzung verstärkt « (Rogge 1995, S. 78). Gestärkt wird dieser Befund durch aktuelle qualitative Biographiestudien. Gewaltbereite Jugendliche werden diesen zufolge durch filmische Gewaltdarstellungen in ihren aggressiven Orientierungen insbesondere dann gestärkt, wenn die medialen präsentierten Gewaltanwendungen gerechtfertigt erscheinen (vgl. Hopf 2001). 
Generell kommunizieren heutige Jugendliche in der Regel einen Wahrnehmungsmodus, der eine eigenständige, generativ gebundene mediale Kompetenz fundiert, die sich deutlich von der der Erwachsenen abgrenzt und stärker intuitiv als kognitiv-emotional geprägt ist (vgl. Vogelsang 2000). Aber auch dieser Befund kann nicht darüber hinweg täuschen, dass die Medienwirkforschung insgesamt noch relativ unterentwickelt ist. Gleichwohl gilt für die die »Alten« und zunehmend auch für die »Neuen Medien«, dass sie für immer mehr Jugendliche zum selbstverständlichen Bestandteil ihres Alltags werden. Medien sind längst keine exklusiven Freizeitgegenstände mehr und vom übrigen Alltag abgesondert, sondern in diesen eingewoben - mit anderen Worten: Medienwelten sind heute in die Lebenswelten von Kindern und Jugendlichen fest integriert.

\subsection{Jugendliche »unter sich« - formelle und informelle Freizeitpraxis}

Autonome, informelle und formell organisierte Gleichaltrigengruppen sind neben gleichund andersgeschlechtlichen Beziehungen und neben Familie, Schule und Arbeitswelt das entscheidende Sozialisationsfeld für Jugendliche. Das Besondere an ihnen ist, dass sie als Orte der Selbstsozialisation Netzwerke für Sozialformen verkörpern, also Topoi, in denen Heranwachsende wesentlich »unter sich» sind (vgl. Mitterauer 1986). Oftmals präsentieren sie in den Biographien der Heranwachsenden die Ersten selbstständig aktivierten sozialen Netze.

Mit Blick auf die quantitative Seite fällt auf, dass im Vergleich zu den 60er-Jahren des 20. Jahrhunderts, wo sich rund die Hälfte der befragten Jugendlichen im Kreis von gleich gesinnten Gleichaltrigen traf, gegenwärtig laut Selbstauskunft zwischen knapp $70 \%$ und $80 \%$ aller Jugendlichen einer vom Wir-Gefühl geprägten Clique angehören - über $90 \%$ der 12- bis 24-jährigen geben sogar an, oft oder sehr oft mit ihren FreundInnen zusammen zu sein (Nolternsting 1998; Der Spiegel 1999, Nr. 28; Jugendwerk der Deutschen Shell 2000). Demgegenüber scheint die Auffassung zu stehen, dass es den Jugendlichen heute an Gemeinsinn fehlt (Oswald 1992; Spiegel Special 1994). Das eigentlich überraschende ist jedoch, dass institutionelle Kontakte nicht etwa im Zuge einer Intensivierung der informellen Beziehungen abnahmen, sondern, zumindest im historischen Vergleich, einen relativ konstanten Level zeigen (vgl. Lüdtke/Pawelka 1989; Jugendwerk der Deutschen Shell 1997). Überholten jedoch bei den informellen »Mitgliedschaften« die Mädchen im Verlauf der 1980er-Jahre die Jungen, ist die Differenz zwischen männlichen und weiblichen Jugendlichen in Bezug auf die Integration in formelle Verbände mit zirka 15 Prozentpunkten relativ unveränderlich geblieben (vgl. Zinnecker 1987; Jugendwerk der Deutschen Shell 1997).

Der zentrale außerhäusliche, formell geregelte Freizeitort für Kinder und Jugendliche ist immer noch der Verein, vor allem der Sportverein. Die stärkere Ausdifferenzierung der von Kindern und Jugendlichen favorisiertesten Sportarten (vgl. Zinnecker 1987, S. 230 f.; vgl. auch Abschnitt 2.2) - unter anderem hat das Interesse für Tischtennis, Tennis, Judo, Skilaufen, Volleyball, Karate, Bodybuilding und tanzbezogene Gymnastik zugenommen deutet an, dass Jugendliche in ihren Freizeitorientierungen heute gebrauchswertorientierter sind und ein dienstleistungsorientiertes, keineswegs mehr ein milieugeprägtes Mitglieds- 
verständnis in und $\mathrm{zu}$ den Vereinen und Verbänden entwickeln. Trotz fortschreitender Technisierung kindlicher und jugendlicher Freizeitwelten kann so nicht von einer »Entkörperlichung « der Freizeitorientierungen in den vergangenen zehn Jahren gesprochen werden - der Körperkult und die ästhetisierende Komponente der gewählten Freizeitbeschäftigung hat sogar an Bedeutung gewonnen. Bei den informellen Kontakten lässt sich allerdings eine Verschiebung von personell und sozial festen peer-Groups und jugendkulturellen Orientierungen hin $\mathrm{zu}$ weicheren und in sich lockeren Szene-Verbindungen erkennen - siehe auch weiter unten. Diese innere Entformalisierung jugendlicher peer-Kontakte findet in Bezug auf die jugendliche Einbindung in formalisierte Kontexte eine Entsprechung in der Abwendung von konventionell-traditionellen Vereinen und Verbänden und einer höheren Präferierung von themengebundenen und temporär begrenzten Organisationsformen wie z. B. Umwelt-, Musik- oder spontanen Hilfs-Initiativen (vgl. Mitterauer 1986; Silbereisen/Vaskovics/Zinnecker 1996).

Eine Palette von quantitativen, insbesondere jedoch qualitativen Studien verdichtet und konkretisiert die Bedeutung von peer-Konstellationen und Gleichaltrigenbeziehungen für Jugendliche. Verschiedene quantitative Studien aus den letzten Jahren heben übereinstimmend hervor, dass keineswegs nur Jugendliche mit einem niedrigen Bildungs- respektive Berufsabschluss an der Verbreiterung der informellen Szenen beteiligt sind. Der Präferenz für informelle Gleichaltrigengruppen generell liegt demnach kein ausgeprägter sozialkultureller Standort in der Gesellschaft zu Grunde. So richtig diese quantitative Trenddiagnose zu dem generellen Anstieg altershomogener jugendlicher Sozialbeziehungen in allen Statusgruppen auch ist, so darf daraus keineswegs rückgeschlossen werden, dass sich die Zusammensetzung der jugendlichen Szenen und peers von den Kriterien sozialer Differenzierung der Gesellschaft völlig abgelöst hat. Qualitative Studien, die verschiedene jugendliche Handlungstypen herauszuarbeiten oder unterschiedliche jugendliche Cliquen dicht zu beschreiben suchten (vgl. u. a. Lenz 1988; Thole 1991), machten deutlich, dass das Kriterium »soziale und ethnische Herkunft« für die Konstituierung von jugendlichen Szenen und peer-groups keineswegs bedeutungslos geworden ist (vgl. aktuell Bohnsack u. a. 1995; Tertilt 1996; Sauter 2000; Eckert/Reis/Wetzstein 2000). Maskulin-, körper- und aktionsorientierte Jugendliche kommen in der Regel aus Familien mit einem niedrigen Sozialstatus. Hingegen rekrutieren sich eher »subjektorientierte« Jugendliche aus Familien mit einem höheren sozialen Status. Gemeinsam ist ihnen aber mit anderen jugendlichen Orientierungen - wie den hedonistischen oder manieristischen, familien- und institutionell gebundenen, den spirituellen oder den alternativ engagierten -, dass sie sich zwar auch noch, aber nicht mehr ausschließlich und primär in festen peers, sondern in offeneren Beziehungsformen bzw. Szenen finden und treffen (vgl. Strzoda 1996; Ferchhoff/Neubauer 1997; Hitzler/Pfadenhauer 2001).

Gegenwärtig ist fast jede der jugend- oder freizeitkulturellen Stilbildungen, die die Jugend zu unterschiedlichen Epochen kreierte, anzutreffen. Neu entwickelte Stile lösen die alten nicht mehr ab, sondern platzieren sich neben diesen und reaktivieren sogar darüber hinaus längst verschwundene jugendkulturelle Muster. Mehr und ausgefeilter denn je wird die Bricolage in jugendkulturellen Szenen als radikalisierte Praxis auf den Ebenen der 
symbolischen Handlungsformen, der Sprachspiele und ästhetischen Codes, der ästhetischen Stilisierungen und Signets, der kulturellen Produktionen, der interaktiven Beziehungsformen und der Selbstinszenierungen dynamisiert und habitualisiert. Dabei widersetzen sie sich zuweilen in einer souveränen, reflexiven Art den An- und Herausforderungen der Medien-, Konsum und Alltagswelt, die bisherige Annahmen der Manipulation und Instrumentalisierung jugendkultureller Kontexte durch die hegemoniale Kultur zynisch konterkariert. Die unkonventionelle Adaption und kreative Dekontextualisierung gesellschaftlicher Vorgaben zu einem szeneeigenen »Symbol- und Zeichensystem ermöglicht (...) eine verläßliche Selbstvergewisserung und tritt in den Dienst der Funktion, Sinn zu verbürgen und $\mathrm{Zu}-$ gehörigkeiten $\mathrm{zu}$ vermitteln «, und erschließt für die Jugendszenen neue Ressourcen der Selbstidentifikationen und -vergewisserung (Vogelsang 2001, S. 286). Interessant ist dabei, dass sich die einzelnen jugendlichen »Bastelsubjekte« zwar in jugendkulturellen Szenen bewegen und sich auch auf diese beziehen, ihre ästhetischen Kompositionen jedoch partiell unabhängig von dem Mainstream dieser Szenen zu realisieren scheinen und ihre stilbildenden, jugendkulturellen Bezugspunkte selbst zu dekontextualisieren wagen. Die Bricolage wird zynisch erweitert zu einem nimmer endenwollenden Sampling, zu einer »remixten « Designkultur, die zynisch und provokant selbst die vermeintlich konsensualen Grundfiguren der gelebten jugendkulturellen Formation kritisch in Frage zu stellen scheint. Das Spiel mit sich Selbst, den eigenen ästhetischen und habituellen Bezugspunkten und den kulturellen Variationen der jeweilen jugendkulturellen Szene scheint zu einem Grundmodus vieler neuer Jugendkulturen geworden zu sein, der es immer schwieriger macht, die Angehörigen einer bestimmten Jugendkultur eindeutig zu identifizieren. Trotz dieses neuen, Eindeutigkeiten vermissenden Remixen innerhalb der einzelnen Jugendkulturen gelingt es W. Ferchhoff und W. Neubauer (1997) immerhin noch, allein 28 differente, zum Teil sich allerdings auch überlappende jugendliche Kulturkontexte und kulturelle Stilformationen auf zu listen. Unter den genannten Stilen finden sich klassische wie die Psycho- und Rockabillys, Skinheads, Rocker und Hippies, aber auch so »exotische« wie die »Funsters«, die postfeministischen Girlies und die »drup-outs« oder »Neuen« wie die Hip-Hop-Bewegten und Apologeten des »Techno«. Trotz der angeführten reflexiven Mentalität scheinen die freizeit- und stilbildenden Jugendkulturen im Vergleich zu ihren historischen Vorgängern an emanzipativen, Dissens und Nonkonformismus betonenden Drive verloren zu haben. Dass die »dissidente Authenzität« der neuen wie neu-alten jugendlichen Freizeit-, Pop- und Rockkulturen jeweils nur noch kurze Zeit anhält, dafür garantieren die Trendsurfer und beobachterInnen, deren Aufgabe und Funktion allein darin liegt, das kreative Potenzial der stylbildenden Jugendmilieus abzuschöpfen, damit es anderorts für konsumfähige Trends kooptiert werden kann - zum einen. Zum anderen zeigen sich Tendenzen der Glorifizierung von eigensinnigen, subversiven und dissidenten Jugendkulturen durch die Milieus und Lebenswelten der Mainstream-Kultur. »Lollapalooza, das schlammige Neo-Woodstock der Piercing-Generation, wurde zum feuchten Traum der Aufsichtsräte und Entertainmentkonzernen. Hocherfreut präsentierte sich der Mainstream nun selbst als Minderheit « (Holert/Terkessidis 1996), was wiederum die kulturelle Avantgarde von kulturellen Minderheitenstilen nicht nur animiert, ihren Stil nun noch abgeschotteter zu kreieren, sondern auch 
dazu, sich auch zum ökonomischen Vermarkter und Reproduzenten »ihrer selbst« zu entwickelten. Und: Mehr den je ist Jugendkultur und jugendliches Freizeitleben nicht mehr nur Adressat von Werbung und Medien, sondern selbst Ort von Werbung und medialer Inszenierung. Jugendkulturen sind heute nicht mehr nur Musikkulturen und Kristalisationspunkte von immer kurzweiligeren Lebensstilen, sondern mehr und mehr auch ästhetisierte Medienorte.

Objekt von Negativschlagzeilen waren in den letzten Jahren insbesondere nationale oder rechtsextreme, xenophobisch oder antisemitisch orientierte jugendliche peer-Gruppen und Szenen. Zwischen 1998 und 1999 hat sich die Anzahl der rechtsextremen, fremdenfeindlichen und antisemitisch motivierten Handlungen und gewalttätigen Straftaten nach Beobachtungen des Verfassungsschutzberichtes von rund 11.000 auf 10.000 reduziert (vgl. Bundesministerium des Inneren 2000, S. 17 f.), um allerdings ein Jahr später wiederum auf 15.951 rechtsextremistisch, fremdenfeindlich und antisemitisch motivierte Straftaten anzusteigen (vgl. Wallraff 2001). Vorliegende regionale Studien zu aggressiven und delinquenten Verhaltensweisen heben bezüglich dieser Befunde jedoch nicht nur hervor, dass sie mit einer erheblichen Dunkelzifferquote belastet sein können, sondern betonen auch die wechselnde Sensibilität der öffentlichen Wahrnehmung als Motor der doch enormen Schwankungen (vgl. Mansel/Hurrelmann 1998; Jansen 2001). Verwiesen wird in diesem Zusammenhang zudem auf die Wirkung der bundesrepublikanischen Massenmedien als situativer »Durchlauferhitzer« fremdenfeindlicher und rechtsnational motivierter Aktionen, zeigt sich doch zumindest in den statistischen Sekundäranalysen eine auffallende Parallelität zwischen der öffentlichen Medienberichterstattung über und dem allgemeinen Anstieg von Gewaltaktionen (vgl. Ohlemacher 1998; kritisch Hopf 2001).

Aktuelle Jugendstudien entdecken in den jugendlichen Selbstauskünften für die letzte Dekade jedoch weder einen bedeutsamen Anstieg antijüdischer und fremdenfeindlicher Vorurteile sowie rechtsnational gefärbter Orientierungen noch einen Anstieg der rechtsextrem motivierten Gewaltakzeptenz (vgl. u. a. Sturzbecher 2001), gleichwohl der Anteil derjenigen Jugendlichen, die xonophobischen und rechtsnational gefärbten Deutungsmustern nahe stehen, sehr hoch ist und unterschiedlichen Studien zufolge bei cirka einem Viertel der Jugendlichen liegt (vgl. u. a. Mansel/Hurrelmann 1998; Münchmeier 2000). Für die Entwicklung fremdenfeindlicher, antisemitischer und rechtsnationaler Orientierungen tragen unterschiedlichen Studien zufolge sowohl desintegrierende Effekte der gesellschaftlichen Modernisierung und die Zunahme von allgemeinen Risikofaktoren Verantwortung als auch familiale Bedingungsfaktoren, subjektiver Anerkennungsmangel und ökonomisch, sozial und kulturell determinierte Ausgrenzungsprozesse. Zu über minderheitenstigmatisierende Deutungen gesteuerte Gewalthandlungen kommt es insbesondere von jugendlichen Cliquen aus benachteiligten Lebenslagen. Die über Selbstdefinitionen habitualisierten dichotomen Bilder von der Welt begünstigen die Gewaltaffinität und die darüber gruppenintern legitimierten gewalttätigen Aktionen. Ihnen kann die Funktion eines Selbstzwecks, eine Reaktion auf Benachteiligungsgefühle ebenso zufallen wie sie der Selbstbehauptung gegenüber anderen oder aber der Absicherung eines ideologischen Konstruktes dienen kön- 
nen (vgl. Bohnsack u. a. 1995; Menschik-Bendele/Ottomeyer 1998; Eckert/Reis/Wetzstein 2000).

Auch wenn zunehmend mehr Mädchen in gewaltorientierten Szenen zu beobachten sind, scheinen weibliche peer-Freundschaften generell gegenüber gleichaltrigen männlichen peer-Gruppen in Bezug auf ihre innere Dynamik eine differente Qualität zu entwickeln. E. Breitenbach und S. Krausträter (1998, S. 400) heben so hervor, dass sich »die Beziehungen oft über Jahre hinweg « entwickeln, »körperlich-zärtliche Komponenten « aufweisen und offen sind für die Bearbeitung der »Unsicherheiten und Probleme mit den Beziehungen zu Jungen «.

Generell auffällig ist die öffentliche Unauffälligkeit vieler jugendlichen Szenen und peer-Beziehungen. Die jugendkulturellen Gleichaltrigengruppen leben in ihrer Mehrzahl eine leise, stille Jugendzeit. Das gesellschaftliche Bild bestimmen neben den exotischen Jugendszenen die expressiven, zu gewalttätigen und kriminalisierbaren Handlungen neigenden Jugendszenen wie die Graffity-Groups, S-Bahnsurfer und Autochrashing-Gangs und Szenen wie die »Psychobillies«. Auf der Suche nach Geborgenheit, Intimität, Anerkennung, Selbstvergewisserung und Autonomie inszenieren sie den Tanz als Kraftprobe, die Politik als Aktion gegen alles Fremde und den Alltag als Rebellion und Randale.

Die Aufmerksamkeit, die informellen jugendlichen Gleichaltrigengruppen und Szenen in den letzten beiden Dekaden immer wieder zukam, können die formellen jugendlichen Gleichaltrigengruppierungen für sich nicht gewinnen. Wird den wenigen hierzu vorliegenden Untersuchungen vertraut, dann sind insbesondere vier unterschiedliche Anspruchshaltungen von Jugendlichen an Jugendverbände zu lokalisieren. Dient der Verband den einen als biographische Karrierestation, so ist er für andere eine Instanz psychosozialer Lebenshilfe, für dritte ein Raum für peer-Beziehungen und für vierte ein Feld zur Entfaltung gesellschaftlichen und sozialen Engagements (vgl. Reichwein/Freund 1992; vgl. auch Jugendwerk der Deutschen Shell 1997). Die traditionellen, nicht sportorientierten Orte formell strukturierter jugendlicher Gleichaltrigenkultur konstituieren sich auch heute noch überwiegend über Prinzipien wie Milieunähe sowie Traditions- und Wertverbundenheit, also über Muster, die nicht nur eine anziehende, sondern aus der Perspektive vieler Jugendlicher auch eine abweisende Botschaft mitteilen. Gegenwärtig zeigen an den klassischen Verbänden - eine Ausnahme bilden hier lediglich die Sportvereine - kaum mehr als $10 \%$ der jeweiligen Heranwachsendengeneration ein konstantes und kontinuierliches Interesse (vgl. Jugendwerk der Deutschen Shell 1992, 1997). Infolge ging auch das statistisch erfasste, aktive ehrenamtliche Engagement der unter 25-jährigen Bevölkerung von 9,1\% im Jahre 1985 auf 6,5 \% im Jahre 1994 zurück (vgl. u. a. Heinze/Strünk 2000). Auch die methodische und konzeptionelle Erweiterung vieler Verbände vermochte bisher keine grundlegende Perspektivenerweiterung zu fundieren, zumindest keine, die deutlich signalisiert, dass zukünftig wieder deutlich mehr Kinder und Jugendliche für die Kinder- und Jugendverbandsarbeit Interesse zeigen. Wenn sich zudem bestätigen sollte, dass die Mitgliedschaft in Sportvereinen keine durchgängig identitätsstabilisierende Wirkung hat, der Alltagsdrogenkonsum von Jugendlichen insbesondere in Fußballvereinen in der Regel höher liegt als bei den nicht vereinsorganisierten Jugendlichen (vgl. Maschler 2001), dann wird die von der 
jeweilige Erwachsenengeneration gewünschte und öffentlich eingeklagte Mitgliedschaft von Jugendlichen in Vereinen zusätzlich infrage gestellt werden.

\section{Zusammenfassung, offene Fragen und Forschungsperspektiven}

\subsection{Zwischen Hoffnungslosigkeit und Euphorie - Resümee}

Trotz vieler offenen Fragen zeigen die Befunde der freizeit- und medienorientierten Jugendforschung, dass in den letzten Jahren vielleicht zu prononciert der Wandel der Kindheits- und Jugendphase betont wurde und darüber strukturelle Kontinuitäten des gesellschaftlichen Modernisierungsprozesses zu sehr im Dunkeln blieben, vielleicht aber auch vor lauter Begeisterung über die Attraktivität modernisierungstheoretischer Begriffe die empirisch gewonnenen Ergebnisse zu schnell subsumtionslogisch verkürzt wurden und werden. Empirische Tiefenbohrungen fallen zuweilen diesem »Schnell-Erkennen-Effekt« zum Opfer. Mit anderen Worten: Der Wandel jugendgenerativer Gestaltungen wird möglicherweise äußerlich von einer ungebrocheneren Kontinuität gestützt als oftmals angenommen. Die vorliegenden Ergebnisse hinsichtlich der von Jugendlichen favorisierten Freizeitformen stützen diesen Befund nachdrücklich. Die Interessen, Freizeithäufigkeiten und die Strukturen der inhaltlichen und quantitativen Gestaltung von jugendlicher Freizeit haben sich überraschend in den letzten Jahrzehnten nur partiell modifiziert. Dieses Ergebnis wird auch nicht durch die Tatsache konterkariert, dass sich die selbstaktiven und damit inhaltlichen Optionen an und in der Freizeit in den letzten zwei Dekaden enorm vervielfältigten. Weiterhin rangieren kreative Freizeittätigkeiten, sportive Praxen und Tanzen sowie die Orientierung auf peers, Cliquen oder Jugendszenen und mit deutlich steigernder Tendenz Urlaubsoptionen auf der Hitliste der bevorzugten Freizeitpräferenzen weit oben. Im Kern findet damit die Prognose von V. Blücher (1956), dass jugendliches Freizeitverhalten durch eine strukturelle Kontinuität gekennzeichnet ist, eine späte, aber nachdrückliche Bestätigung. Dennoch signalisieren die einzelnen Freizeitpraxen eine deutlich ausgeprägte Vervielfältigung und Ausdifferenzierung und eine Erweiterung insofern, als dass der Umgang mit Medien und der Konsum von medialen Angeboten zugenommen hat und auf dem Weg $\mathrm{zu}$ einem integralen Bestandteil jugendlichen Freizeitverhaltens ist. Insgesamt weisen die Freizeitoptionen von Jugendlichen seit dem letzten Viertel des letzten Jahrhunderts einen hochdifferenzierten Geschmacks-, Ausgestaltungs- und Stilrichtungspluralismus auf. Die in den verschiedenen Studien dokumentierte inhaltliche Ausdifferenzierung kann einerseits auf eine gewendete, deutlich »jugendzentriertere« Umfragetechnik zurückzuführen sein, aber auch andererseits eine reale Pluralisierung und Ausfächerung jugendlicher Optionen für einzelne Freizeitformen spiegeln. Hinter der vordergründigen Fassade einer Vielfalt »frei wählbarer « sozialkultureller Optionen an die Freizeit bestehen jedoch alte schichtund geschlechtsspezifische Ungleichheiten in neuem Gewande fort - und: Die sozialkulturellen Praxen der Jugend an der Schwelle zu Beginn des neuen Jahrtausend sind nicht nur »maßgeschneidert und dennoch von der Stange«, sondern auch nicht mehr für den gesamten Weg durch die Jugendbiographie entworfen. 
Nicht nur die individuellen Optionen für diese oder jene Freizeitmöglichkeit haben sich potenziert, insgesamt haben sich die Fahrpläne durch die Jugendbiographie in den vergangenen zwei Dekaden aufgeweicht. Der Weg durch die Jugendzeit hat in den 80er-Jahren des 20. Jahrhunderts endgültig das rechtlich und gesellschaftlich betonierte Flussbett überschwemmt und neue Verläufe gesucht und gefunden. Neben der jugendlichen Orientierung an starre Altersnormen suchen Jugendliche ihren Weg in der Balance zwischen Schule und Clique sowie zwischen individueller und institutionell vorgegebenen Zeitrahmungen oder aber in der Abkehr von klassischen zeitlichen Vorstrukturierungen und in der frühen Einkehr in ein neues familiales Dach (vgl. Fuchs-Heinritz/Krüger 1991; Abels 1993; Ferchhoff/Neubauer 1997). Die zunehmend komplexer, mobiler und undurchschaubarer werdende Gesellschaft hat viele Jugendliche in den 90er-Jahren des letzten Jahrhunderts eine »Patchwork-Identität« entwickeln lassen (vgl. Keupp 1992; Keupp u. a. 1999), mit der sie situativ heterogene Anforderungen $\mathrm{zu}$ »meistern« suchen, ohne Garantie, dies auch zu schaffen. Ob sie dabei mehrheitlich auf pragmatische, idealistische, karrieristische oder spaß- und lustbetonte, systemkonforme oder nonkonforme Lebensbewältigungsstrategien verlassen, bleibt zu beobachten. Der Weg durch die Jugendbiographie ist zu einer schwierigen, holprigen Tour - für viele sogar zu einer Tortur - geworden, auch, weil Jugendliche heute ein dynamisches »Abschleifen traditioneller Sozialformen« (Ziehe 1994, S. 259) erleben. Jugendlichen stehen kaum noch bindende und verlässliche Sicherheiten zur Verfügung, auf die sie ritualisiert zur Bewältigung von Risiken des Alltags zurückgegriffen können, auf die - quasi als ritualisiertes soziales Korsett - bei der Herstellung und Aufrechterhaltung von sozialen Kontakten Verlass ist.

Die Involviertheit in Freizeitnetze, die subjektiven Freizeitpräferenzen sowie die autonom gestaltbaren Orte jugendlicher Selbstsozialisation haben bezüglich der biografischen Lebenswegoptionen gegenüber den klassischen Sozialisationskontexten an Bedeutung gewonnen und diesen viele ihrer lebenslaufprägenden Relevanzen beraubt. Freizeit ist nicht nur nach wie vor ein bedeutendes Sozialisations- und Moratoriumsfeld für Jugendliche, sondern auch ein entscheidendes Distinktions- und Identifikationsfeld (vgl. Fritzsche 1997, S. 348) - dies wird durch die aktuellen Freizeit- und Medienstudien nachhaltig untermauert.

\subsection{Offene Forschungsfragen und -zugänge}

Die Rekonstruktionen jugendgeprägter gesellschaftlicher Wirklichkeit haben sich in der zurückliegenden Dekade im Wesentlichen auf eine modernisierungstheoretische Perspektive eingependelt. Alles differenziert sich aus, ist vielfältiger und in sich pluraler als je zuvor, bunter und uneindeutiger, standardisierter und zugleich mehr denn je entstardardisiert, zumindest anders als früher und doch, folgen wir den vorliegenden Befunden und die sie codierenden modernisierungstheoretischen Vokabeln, strukturell vom Tradierten geprägt. Ein kritisches Gegenlesen dieser Ortsbestimmung der gesellschaftlichen Situation ist kaum noch zu beobachten. Nicht nur, aber auch weil das Basisvokabular dieser Perspektive - Individualisierung, Pluralisierung und Ausdifferenzierung - inzwischen zum Standardrepertoire der AktorInnen gesellschaftsreflektierender Alltagsgespräche gehört, ist Skepsis ge- 
genüber dieser anscheinend omnipotenten Theorieperspektive angebracht und zu Fragen, ob nicht gerade ihre Allgegenwart sie ihres aufklärerischen Stachels beraubt. Zumindest ist darüber nach zu denken, ob pauschale Hinweise auf gesellschaftliche Individualisierungs-, Pluralisierungs- und Entstandardisierungstendenzen ausreichen, den Wandel von Kindheit und Jugend genau zu markieren und die Spezifika der heutigen Kindheit und Jugend zu beschreiben. Diese Frage fordert natürlich auch dazu auf, zentrale Aussagen der vorgetragenen Befunde und Wertungen zu den jugendlichen Freizeit- und Medienorientierungen zu überdenken und die zukünftige Jugendfreizeit- und Jugendmedienforschung neu zu justieren. Die aktuell favorisierten modernisierungstheoretisch angelegten Theorieperspektiven sind so möglicherweise um deutlich akzentuierte kulturtheoretische Ortsbesimmungen sowie um sozialökologische, subjekt- und biographietheoretische Ansätze aufzustocken, damit die Dialektik von gesellschaftlichen, lebenslagen und -weltlichen sowie ökologischen beziehungsweise sozialräumlichen Bedingungen und individuellen Prozessen in ihrer ambivalenten Dynamik noch präziser erfasst werden kann. Unabhängig von der konkreten Ausformulierung einer für empirische Forschungen offenen Theorie jugendlichen Freizeitund Medienverhaltens und der generellen Forderung, freizeit- und medienbezogene Fragestellungen und Forschungen wieder stärker aufeinander zu beziehen und zu verkoppeln, sind folgende Forschungsdesiderate identifizierbar:

- Wünschenswert erscheinen quantitative Studien zum Freizeit- und Medienverhalten, die modernisierungstheoretische und kultursoziologische Annahmen und Behauptungen empirisch zu überprüfen suchen. Entsprechende Forschungsvorhaben hätten insbesondere auch den Blick auf soziale, kulturelle, ethnische und geschlechtsspezifische Ungleichheiten im Forschungsprofil zu lenken.

- Bislang zu wenig Beachtung wurde den Freizeitwelten und -ansprüchen von weiblichen Jugendlichen und Heranwachsenden und ihren zu männlichen Kommunikationsformen differenten Beziehungs- und Freundschaftsansprïchen und -varianten geschenkt.

- Qualitative Untersuchungen zur biographischen Bedeutung der in der Adoleszenz gewählten Freizeit- und Medienpräferenzen fehlen bislang ebenso wie tiefer fragende Studien zur Rekonstruktion der sinnhaften Bedeutung, die Jugendliche ihren Freizeit- und Medienaktivitäten zuschreiben.

- Fernab expressiver Stilbildungen und die gesellschaftlichen Konventionen sprengender Freizeitpraxen sich verortende jugendliche Freizeitgestaltungen geraten immer noch zu selten in den Focus der forschenden Interessen. Zwar ist die Verteilung jugendlichen Freizeitverhaltens hinlänglich dokumentiert, jedoch die an diese Orientierungen gekoppelten Wünsche, Bedürfnisse und Hoffnungen, also quasi die innere Dynamik der ausgeübten Freizeittätigkeiten ist weitgehend noch eine »Black Box«. Mit anderen Worten: Die qualitative Seite der Freizeitwelten der »Stinkies« und »Normalos« ist bisher nur wenig erforscht.

- Unerforscht ist auch noch die Bildungs- und Freizeitwelt der außerschulischen, institutionalisierten Angebote. Die empirische Deskription und Rekonstruktion der Bedeutung und »Wirkung« außerschulischer, institutionalisierter Freizeitwelten - beispielsweise der Kinder- und Jugendarbeit - steht noch aus.

- Von Interesse sind biographische Studien, die nach Kontinuität oder Diskontinuität von Freizeitverhalten in Lebensgeschichten fragen oder nach der biographischen Bedeutung von Freizeit- und Medienkarrieren für spätere Berufswege. 
- Kaum durchgeführt wurden bislang kultur- und ideologiekritische Analysen der ProduzentInnenseite: Inwieweit präformieren Freizeitangebote und -märkte das Freizeit- und Medienverhalten von Jugendlichen bewusst und über welches Wissen verfügen die professionellen »FreizeitmacherInnen « von ihren BesucherInnen und KonsumentInnen.

- Unbeobachtet blieben bisher die neuen Freizeitformen der Wissensgesellschaft. Ihre Relevanz in Bezug auf die Initiierung von Bildungsprozessen und hinsichtlich ihrer Bedeutung bezüglich der Steuerung gesellschaftlicher Selektionsprozessse ist noch weitgehend unbekannt. Die sozialisatorischen Bedeutung und Wirkung digitaler Welten und der Fluchten in die »sozialen Labatorien « (Marotzki 1997) des Cyberspace zu eruieren, oblieg zukünftigen Studien.

- Weitgehend unbeleuchtet sind aus sozial- und erziehungswissenschaftlicher Perspektive bislang auch noch die neuronalen Aspekte der Medienrezeption. Diesbezüglich scheinen interdisziplinär angelegte Forschungsvorhaben sinnvoll und notwendig.

Mit Blick auf die vorliegenden Panorama- und Meinungsstudien ist zu hoffen, dass künftige Forschungsprojekte ihre Ergebnisse weniger theoriefern publizieren und neue Fragen so methodisch operationalisieren, dass empirische Erkenntnisse nicht nur theorierezipierend, sondern auch theoriekorrigierend und theoriegenerierend auswertbar werden. Hierzu erscheint es sinnvoll, sich von monolithischen, einseitig quantitative oder qualitative Methoden favorisierenden methodologischen Grundlegungen $\mathrm{zu}$ verabschieden und noch stärker als bisher methodentriangulierend vor zu gehen. Um einen mehrperspektivischen Zugang zur Realität jugendlichen Freizeit- und Medienverhaltens zu gewinnen, ist einer Kombination von qualitativen und quantitativen Methoden den Vorzug zu geben. Erst die Ausformulierung eines solchen umfangreichen, aber in sich ausdifferenzierten und nicht in Beliebigkeit versinkenden Theorie- und Methodenpluralismus könnte den Bezugsrahmen für die Durchführung von Untersuchungen abgeben, die die skizzierten Forschungsdefizite im Bereich der Jugendfreizeit- und -medienforschung empirisch und theoretisch aufzuarbeiten suchen.

Wenn den öffentlichen Gesprächen vertraut wird, dann kennt die ErwachsenenGesellschaft »die« nachwachsende Generation. Über Jugend - insbesondere über deren Freizeitgewohnheiten und Medienverhalten - meint heute ein jeder mitreden zu können. Das Forschungswissen, egal wie dieses im Einzelnen auch bewertet wird, spielt in den öffentlichen Diskussionen kaum eine Rolle. Hier scheint die Jugendforschung angeregt, ihre Präsentationsweisen und -formen zu überdenken. Gleichwohl gilt: Mögen öffentliche Bilder und Muster noch so stringent und nachvollziehbar ihre Zeitdiagnose vom Zustand der Jugend vortragen, letztendlich bleibt es konkreten, empirischen Studien und Arbeiten vorbehalten, sensibel darüber aufzuklären, wie sich die Jugendphase in ihrer ambivalenten Dynamik gestaltet. Wer hofft, das Wissen über jugendliches Freizeit- und Medienverhalten im virtuellen Netz unter »www.jugendfreizeitmedien.de« gut verpackt und aufbereitet zu finden, unterliegt einer Illusion.

\section{Literatur}

Abels, H.: Jugend vor der Moderne. Opladen 1993.

Baacke, D.: Der sozialökologische Ansatz. In: deutsche jugend, 38. Jg. (1980), Heft 11, S. 493-505. 
Baacke, D./Ferchhoff, W.: Jugend und Kultur. In: Krüger, H.-H. (Hrsg.): Handbuch der Jugendforschung. Opladen 1992, S. 403-444.

Baacke, D./Sander, U./Vollbrecht, R.: Lebenswelten Medienwelten. Opladen 1990.

Backhaus-Starost, A./Backhaus, E.: Freizeitaktivitäten von Arbeiterjugendlichen. Frankfurt a. M. 1975.

Bals, Ch.: Halbstarke unter sich. Köln u. Berlin 1962.

Barthelmes, J./Sander, E.: Medien in Familie und Peer-group. Opladen 1997.

Bäumler, Ch./Banger, M./Schwab, U.: Kirche - Clique - Religion. Weinheim u. München 1994.

Beck, U.: Risikogesellschaft. Auf dem Weg in eine andere Moderne. Frankfurt a. M. 1986.

Beck, U.: Die Zukunft von Arbeit und Demokratie. Frankfurt a. M. 2000.

Beck, U./Bonß, W.: Soziologie und Modernisierung. In: Soziale Welt, 35. Jg. (1984), S. 381-406.

Beck, U./Beck-Gernsheim, E. (Hrsg.): Riskante Freiheiten. Individualisierung in modernen Gesellschaften. Frankfurt a. M. 1994.

Becker, H. u. a.: Pfadfinderheim, Teestube, Straßenleben. Jugendliche Cliquen und ihre Sozialräume. Frankfurt a. M. 1984.

Behnken, I. u. a.: Schülerstudie '90. Jugendliche im Prozess der Vereinigung. Weinheim u. München 1991.

Bloch, E.: Prinzip Hoffnung. Gesamtausgabe. Bd. 5. Frankfurt a. M. 1977.

Blücher, V. Graf: Freizeit in der Industriellen Gesellschaft. Stuttgart 1956.

Blücher, V. Graf: Die Generation der Unbefangenen. Düsseldorf u. Köln 1966.

Blücher, V. Graf: Jugend, Bildung, Freizeit. Dritte Untersuchung zur Situation der deutschen Jugend (EMNID-STUDIE). Bielefeld 1967.

Bohnsack, R. u. a.: Die Suche nach Gemeinsamkeit und die Gewalt der Gruppe. Opladen 1995.

Böpple, F./Knüfer, R.: Generation XTC. München 1996.

Bourdieu, P./Wacquant, L.: Schöne neue Begriffswelt. In: Le Monde diplomatique, Mai 2000, S. 7.

Bourdieu, P.: Die feinen Unterschiede. Frankfurt a. M. 1984.

Bourdieu, P. u a.: Das Elend der Welt. Konstanz 1997.

Bourdieu, P.: Über das Fernsehen. Frankfurt a. M. 1998.

Braddock, K./Hertel, W.: Völlig losgelöst. Bürgerliche und musikalische Konventionen sind ihnen schnuppe. In: musikexpress, Ausgabe März 2001, S. 27.

Bravo-Studie: Jugend, die kaufbeeinflussende Kraft in der Familie. München 1986.

Breitenbach, E./Kausträter, S.: »Ich finde, man braucht irgendwie eine Freundin«. In: Zeitschrift für Sozialisationsforschung und Soziologie der Erziehung, 18. Jg. (1998), Heft 4, S. 389-402.

Brinkhoff, K. P.: Sport und Sozialisation im Jugendalter. Weinheim u. München1998.

Bronfenbrenner, W.: Ökologische Sozialisationsforschung. Stuttgart 1976.

Büchner, P: Aufwachsen in den 80er-Jahren. In: Büchner, P./Krüger, H.-H./Chisholm, L. (Hrsg.): Kindheit und Jugend im interkulturellen Vergleich. Opladen 1990, S. 79-93.

Bundesministerium des Innern (Hrsg.): Verfassungsschutzbericht 1999. Fulda 2000.

Clarke, J. u. a.: Jugendkultur als Widerstand. Frankfurt a. M. 1979.

Cloos, P.: Jugend als Avantgarde ihrer eigenen Abschaffung? In: Sozialwissenschaftliche Literatur Rundschau, 21. Jg. (1998), Heft 36, S. 37-54.

DE:BUG: Zeitschrift für Musik, Medien, Kultur und Selbstbeherrschung. Berlin 2001.

Deese, U. u. a.: Jugend und Jugendmacher. München u. Düsseldorf 1996.

Dehn, G.: Proletarische Jugend. Lebensgestaltung und Gedankenwelt der großstädtischen Proletarierjugend. Berlin 1929.

Deinet, U.: Sozialräumliche Jugendarbeit. Opladen 1999.

Der Spiegel: Boygroups sind das Ding der Neunziger. 1997, Heft Nr. 6, S. 183-188.

Der Spiegel: Eltern ohne Einfluss. Ist Erziehung sinnlos? 1998, Heft Nr. 47.

Der Spiegel: Die Jungen Milden. 1999, Heft Nr. 28, S. 94-103.

Dinse, R.: Das Freizeitleben der Großstadtjugend. 5000 Jungen und Mädchen berichten. Berlin 1932.

Dörrie, D.: Das Trockenshampoo. In: Süddeutsche Zeitung, 22.02.2001, S. 19.

Eberle, Th. S.: Ethnomethodologische Konversationsanalyse. In: Hitzler, R./Honer, A. (Hrsg.): Sozialwissenschaftliche Hermeneutik. Opladen 1997, S. 245-280. 
Eberle, Th.: Motivation des Fernsehverhaltens Jugendlicher. Heilbrunn 2000.

Eckert, R. u. a.: Sinnwelt Freizeit. Jugendliche zwischen Märkten und Verbänden. Opladen 1991.

Eckert, R./Reis, Ch./Wetzstein, Th.: »Ich will halt anders sein wie die anderen«. Opladen 2000.

Fend, H.: Entwicklungspsychologie des Jugendalters. Opladen 2000.

Ferchhoff, W./Neubauer, G.: Patchwork-Jugend. Eine Einführung in postmoderne Sichtweisen. Opladen 1997.

Fischer, A./Münchmeier, R.: Die gesellschaftliche Krise hat die Jugend erreicht. In: Jugendwerk der Deutschen Shell (Hrsg.): Jugend '97 - Zukunftsperspektiven, Gesellschaftliches Engagement, Politische Orientierungen. Opladen 1997, S. 11-25.

Friebel, H. u. a.: Selbstorganisierte Jugendgruppen zwischen Partykultur und politischer Partizipation. Opladen 1979.

Fritzsche, Y.: Jugendkulturen und Freizeitpräferenzen. In: Jugendwerk der Deutschen Shell (Hrsg.): Jugend '97. Opladen 1997, S. 343-378.

Fritzsche, Y.: Modernes Leben - Gewandelt, vernetzt und verkabelt. In: Jugendwerk der Deutschen Shell (Hrsg.): Jugend 2000 - 13. Shell Jugendstudie. Opladen 2000, S. 181-221.

Fuchs, W.: Entspannung im Alltag. In: Jugendwerk der Deutschen Shell (Hrsg.): Jugendliche und Erwachsene '85. Generationen im Vergleich, Bd. 2. Opladen 1985, S. 7-34.

Fuchs, W./Zinnecker, J.: Nachkriegsjugend und Jugend heute. Werkstattbericht aus einer laufenden Studie. In: Zeitschrift für Sozialisationsforschungund Soziologie der Erziehung 5. Jg., (1985), Heft 1, S. 5-28.

Fuchs-Heinritz, W./Krüger, H.-H.: Feste Fahrpläne durch die Jugendphase. Opladen 1991.

Geene, St.: Death disco Club. In: spex. Das Magazin für Popkultur. 2001, Nr. 3, S. 60.

Grieswelle, D.: Jugend und Freizeit. Bedingungen außerschulische Jugendarbeit. München 1978.

Groebel, J.: Ergebnisse der internationalen UNESCO-Studie »Jugend und Gewalt«. In: Roters, G./Klingler, W./Gerhards, M. (Hrsg.): Mediensozialisation und Medienverantwortung. Baden-Baden 1999, S. 99-112.

Harberg, C.: Früh übt sich: Nur mit Handy echt fett. In: Commerzbank Journal, 14. Jg. (2000), Heft 1, S. 3639.

Heinze, R. G./Strünck, Ch.: Die Verzinsung des sozialen Kapitals. Freiwilliges Engagement im Strukturwandel. In: Beck, U. (Hrsg.): Die Zukunft von Arbeit und Demokratie. Frankfurt a. M. 2000, S. 171-216.

Heitmeyer, W./Peter, H.: Jugendliche Fußballfans. Weinheim u. München 1988.

Heitmeyer, W./Olk, Th.: Das Individualisierungs-Theorem. Bedeutung für die Vergesellschaftung von Jugendlichen. In: Heitmeyer, W./Olk, Th. (Hrsg.): Individualisierung von Jugend. Weinheim u. München 1990, S. 11-34.

Hitzler, R./Bucher, Th./Niederbacher, A.: Leben in Szenen. Kulturen unter den Bedingungen der Spätmoderne. Opladen 2001.

Hitzler, R./Pfadenhauer, M.: Techno-Soziologie. Erkundungen einer Jugendkultur. Opladen 2001.

Hitzler, R./Honer, A. (Hrsg.): Sozialwissenschaftliche Hermeneutik. Opladen 1997.

Holert, T./Terkessidis, M.: Einführung in den Mainstream der Minderheiten. In: Holert, T./Terkessidis, M. (Hrsg.): Mainstream der Minderheiten. Berlin 1996, S. 5-19.

Hopf, Ch.: Gewalt, Biographie, Medien. In: Zeitschrift für Sozialisationsforschung und Soziologie der Erziehung, 21. Jg. (2001), Heft 2, S. 150-169.

Horkheimer, M./Adorno, Th. W.: Dialektik der Aufklärung. Frankfurt a. M. 1969.

Hübner-Funk, S. u. a.: Sozialisation und Umwelt. Berufliche Orientierungen und Gesellschaftsformen von Hauptschülern im sozialökologischen Kontext. München 1983.

Husserl, E.: Zur Phänomenologie der Intersubjektivität. Hamburg 1973.

Jaide, W.: Generationen eines Jahrhunderts. Opladen 1988.

Jansen, F.: Die Polizei registriert für 2000 Anstieg um knapp 4000 Delikte. In: Der Tagesspiegel - online, 17. Januar 2001.

Jugendwerk der Deutschen Shell (Hrsg.): Jugend zwischen 15 und 24. Bielefeld 1955.

Jugendwerk der Deutschen Shell (Hrsg.): Jugend - Bildung und Freizeit. Hamburg u. Münster 1966.

Jugendwerk der Deutschen Shell (Hrsg.): Jugend zwischen 13 und 24. Hamburg u. Münster 1975.

Jugendwerk der Deutschen Shell (Hrsg.): Jugend und Erwachsene '85. 5 Bde. Opladen 1985.

Jugendwerk der Deutschen Shell (Hrsg.): Shell-Studie 1992 - Jugend '92. 4 Bde. Opladen 1992. 
Jugendwerk der Deutschen Shell (Hrsg.): Shell-Studie 1997 - Jugend '97. Opladen 1997.

Jugendwerk der Deutschen Shell (Hrsg.): Jugend 2000 - 13. Shell Jugendstudie. 2 Bde. Opladen 2000.

Juice - HipHop, Rhythm, Beatz, Graffiti: Ausgabe März 2001.

Keupp, H.: Verunsicherungen. Risiken und Chancen des Subjekts in der Postmoderne. In: Rauschenbach, Th./Gängler, H. (Hrsg.): Soziale Arbeit und Erziehung in der Risikogesellschaft. Neuwied 1992, S. 165185.

Keupp, H. u. a.: Identitätskonstruktionen. Das Patchwork der Identitäten in der Spätmoderne. Reinbek b. Hamburg 1999.

Kieper, M.: Lebenswelten verwahrloster Mädchen. München 1980.

Klingler, W./Roters, G./Gerhards, M. (Hrsg.): Medienrezeption seit 1945. Baden-Baden 1999.

Kofler, G./Graf, G. (Hrsg.): Sündenbock Fernsehen? Berlin 1995.

Kolip, P.: Tabak- und Alkoholkonsum bei Jugendlichen. In: Leppin, A./Hurrelmann, K./Petermann, H. (Hrsg.): Jugendliche und Alltagsdrogen. Neuwied u. Krieftel 2000, S. 24-44.

Krotz, F.: Computervermittelte Medien. In: Roters, G./Klingler, W./Gerhards, M. (Hrsg.): Mediensozialisation und Medienverantwortung. Baden-Baden 1999, S. 155-172.

Krüger, H.-H.: Zum Wandel von Freizeitverhalten und kulturellen Lebensstilen bei Heranwachsenen in Westdeutschland. In: Büchner, P./Krüger, H.-H. (Hrsg.): Aufwachsen hüben und drüben. Opladen 1991, S. 203-223.

Küster, E. U.: Fremdes Gelände. Ethnographie eines Jugendhauses. Münster 2001.

Lenz, K.: Die vielen Gesichter der Jugend. Frankfurt a. Main 1988.

Leppin, A./Hurrelmann, K./Petermann, H. (Hrsg.): Jugendliche und Alltagsdrogen. Neuwied u. Krieftel 2000 .

Lessing, H./Liebel, M.: Jugend in der Klassengesellschaft. Marxistische Jugendforschung und antikapitalistische Jugendarbeit. München 1974.

Luca, R.: Weibliche Identitätsentwicklung und mediale Vorbilder. In: Friebertshäuser, B./Jakob, G./KleesMöller, R. (Hrsg.): Sozialpädagogik im Blick der Frauenforschung. Weinheim 1997, S. 83-94.

Lüdtke, H./Grauer, G.: Jugend - Freizeit - Offene Tür. Methoden und Daten der empirischen Erhebung in Jugendfreizeitheimen. Weinheim u. Basel 1972.

Lüdtke, H.: Jugendliche in ihrer Freizeit. Interessen und Verhalten. In: Markefka, M./Nave-Herz, R. (Hrsg.): Handbuch der Familien- und Jugendforschung, Bd. 2. Neuwied u. Frankfurt a. M. 1989, S. 571-588.

Lüdtke, H./Pawelka, A.: Jugend in Organisationen. In: Markefka, M./Nave-Herz, R. (Hrsg.): Handbuch der Familien- und Jugendforschung, Bd. 2, Neuwied u. Frankfurt a. Main 1989, S. 571-588.

Lukesch, H.: Das Forschungsfeld »Mediensozialisation« - eine Übersicht. In: Roters, G./Klingler, W./Gerhards, M. (Hrsg.): Mediensozialisation und Medienverantwortung. Baden-Baden 1999, S. 59-83.

Mansel, J./Hurrelmann, K.: Aggressives und delinquentes Verhalten Jugendlicher im Zeitvergleich. In: Kölner Zeitschrift für Soziologie und Sozialpsychologie, 50. Jg. (1998), Heft 1, S. 78-109.

Maschler, N.: Der Sportler trinkt gerne. In: die tageszeitung, 6.03.2001, S. 7.

Matthesius, B.: Anti Sozialfront. Opladen 1992.

Menschik-Bendele, J./Ottomeyer, K.: Sozialpsychologie des Rechtsextremismus. Opladen 1998.

Meyer, E.: Die Techno-Szene. Opladen 2000.

Mitterauer, M.: Sozialgeschichte der Jugend. Frankfurt a. Main 1986.

Müller, H.: Zum Drogenkonsum bei der Schuljugend in den neuen Bundesländern. In: Leppin, A./Hurrelmann, K./Petermann, H. (Hrsg.): Jugendliche und Alltagsdrogen. Neuwied u. Krieftel 2000, S. 45-63.

Müller, I.: Jugend und Freizeit. Aufgabe der allgemeinen Sozialisation oder einer intentionalen Erziehung durch die Schule. Frankfurt a. M. 1984.

Müller, B./Rosenow, R./Wagner, M.: Dorfjugend Ost - Dorfjugend West. Freiburg i. Br. 1994.

Münchmeier, R. (Hrsg.): Miteinander - Nebeneinander - Gegeneinander? In: Jugend 2000 - 13. Shell Jugendstudie. Opladen 2000, S. 221-259.

Musikexpress: Ausgabe März 2001.

Negt, O.: Lebendige Arbeit, enteignete Zeit. Frankfurt a. M. u. New York 1984. 
Neumann-Braun, K./Deppermann, A.: Ethnographie und Kommunikationskulturen Jugendlicher. In: Zeitschrift für Soziologie, 27. Jg. (1998), Heft 4, S. 239-255.

Noelle-Neumann, E.: Die Verteidigung des Lesens - Kann man einen langfristigen Trend mit der Sozialforschung wieder umdrehen? In: Klingler, W./Roters, G./Gerhards, M. (Hrsg.): Medienrezeption seit 1945. Baden-Baden 1999, S. 13-26.

Nolteernsting, E.: Jugend. Freizeit. Geschlecht. Opladen 1998.

Oevermann, U.: Zur Sache. In: Friedeburg, L./Habermas, J. (Hrsg.): Adorno-Konferenz 1983. Frankfurt a. M. 1983.

Ohlemacher, Th.: Fremdenfeindlichkeit und Rechtsextremismus. In: Soziale Welt, 49. Jg. (1998), Heft 4, S. 319-332.

Opaschowski, H. $\left({ }^{2} 1996\right)$ : Tourismus - eine systematische Einführung. Opladen.

Onna, B. van: Jugend und Vergesellschaftung. Eine Auseinandersetzung mit der Jugendsoziologie. Frankfurt a. M. 1976.

Oswald, H.: Beziehungen zu Gleichaltrigen. In: Jugendwerk der Deutschen Shell (Hrsg.): Jugend '92. Opladen 1992, S. 319-333.

Paus-Haase, I. u. a.: Talkshows im Alltag von Jugendlichen. Opladen 1999.

Peukert, D.: Jugend zwischen Krieg und Krise. Lebenswelten von Arbeiterjungen in der Weimarer Republik. Köln 1987.

Projektgruppe Jugendbüro: Die Lebenswelt von Hauptschülern. München 1973.

Projektgruppe Jugendbüro: Subkultur und Familie als Orientierungsmuster. München 1977.

Radde, M./Sander, U./Vollbrecht, R. (Hrsg.): Jugendzeit - Medienzeit. Weinheim u. München 1988.

Reichwein, S./Freund, Th.: Karrieren, Action, Lebenshilfe. Opladen 1992.

Rieker, P.: Ethnozentrismus im Jugendalter. In: Zeitschrift für Sozialisationsforschung und Soziologie der Erziehung, 20. Jg. (2000); Heft 1, S. 39-54.

Rogge, J.-U.: Die Faszination und die Bedeutung medialer Gewalt aus Sicht von Heranwachsenden. In: Kofler, G./Graf, G. (Hrsg.): Sündenbock Fernsehen? Berlin 1995, S. 55-80.

Rose, L.: Abenteuer - nur für Jungen? In: Friebertshäuser, B./Jakob, G./Klees-Möller, R. (Hrsg.): Sozialpädagogik im Blick der Frauenforschung. Weinheim 1997, S. 171-182.

Rosenmayr, L.: Familie und Freizeitgewohnheiten jugendlicher Arbeiter. Wien 1963.

Rosenmayr, L./Köckeis, E./Kreutz, H.: Kulturelle Interessen von Jugendlichen. Wien 1966.

Roters, G./Klingler, W./Gerhards, M. (Hrsg.): Mediensozialisation und Medienverantwortung. Baden-Baden 1999.

Sauter, S.: Wir sind »Frankfurter Türken«. Adoleszente Ablösungsprozesse in der deutschen Einwanderungsgesellschaft. Frankfurt a. M. 2000.

Sander, U./Vollbrecht, R.: Zwischen Kindheit und Jugend. Weinheim und München 1985.

Sauter, S.: Wir sind »Frankfurter Türken«. Adoleszente Ablösungsprozesse in der deutschen Einwanderungsgesellschaft. Frankfurt a. M. 2000.

Schäffer, B.: Die Band. Opladen 1996.

Scharmann, D.-L.: Konsumverhalten von Jugendlichen. München 1965.

Schmidt, J.: Jugendtypen aus dem Arbeitermilieu. Ein Beitrag zur Typologie der erwerbstätigen Jugend. Weimar 1934.

Schmidt, T.: Punk sein, Techno machen. In: DE:BUG, elektronische Lebensaspekte, 2001, Heft 44, S. 20.

Schorb, B./Mohn, E./Theunert, H.: Sozialisation durch (Massen-)Medien. In: Hurrelmann, K./Ulich, D. (Hrsg.): Neues Handbuch der Sozialisationsforschung. Weinheim u. Basel 1991.

Schröer, N.: Umriss einer hermeneutischen Wissenssoziologie. In: Schröer, N. (Hrsg.): Interpretative Sozialforschung. Opladen 1994.

Schultz, C.: Die Halbstarken. Hamburg 1912.

Schulze, G.: Spontangruppen der Jugend. In: Markefka, M./Nave-Herz, R. (Hrsg.): Handbuch der Familienund Jugendforschung, Bd. 2. Neuwied u. Frankfurt a. M. 1989, S. 553-569.

Silbereisen, R. K./Vaskovics, L. A./Zinnecker, J. (Hrsg.): Jungsein in Deutschland. Jugendliche und junge Erwachsene 1991 und 1996. Opladen 1996.

Spiegel-Special: Die Eigensinnigen. Selbstporträt einer Generation. November 1994. 
SPoKK (Hrsg.): Kursbuch Jugendkultur. Stile, Szenen und Identitäten vor der Jahrtausendwende. Mannheim 1997.

SPoKK (Hrsg.): Kursbuch Jugendkultur. Mannheim 1997.

Strzelewicz, W.: Jugend in freier Zeit. München 1965.

Strzoda, C.: Freizeitverhalten und Freizeitmuster. In: Silbereisen, R. K./Vaskovics, L. A./Zinnecker, J. (Hrsg.): Jungsein in Deutschland. Jugendliche und junge Erwachsene 1991 und 1996. Opladen 1996, S. 261-280.

Strzoda, C./Zinnecker, J.: Das persönliche Zeitbudget zwischen 13 und 30. In: Silbereisen, R. K./Vaskovics, L. A./Zinnecker, J. (Hrsg.): Jungsein in Deutschland. Jugendliche und junge Erwachsene 1991 und 1996. Opladen 1996, S. 281-300.

Sturzbecher, D. (Hrsg.): Jugend in Ostdeutschland. Opladen 2000.

TAZ: Wir liegen konsequent daneben. In: die tageszeitung, 27.06.1997, S. 13.

Tenbruck, F. H.: Jugend und Gesellschaft. Freiburg 1962.

Tertilt, H.: Turkish Power Boys. Frankfurt a. M. 1996.

Thole, W.: Familie - Szene - Jugendhaus. Alltag und Subjektivität einer Jugendclique. Opladen 1991.

Thole, W./Müller, B.: Konstitution und Dynamik der sozialpädagogischen Kinder- und Jugendarbeit. Hildesheim u. Kassel 2001 (MS).

Tippelt, R. u. a.: Jugend und Medien. Weinheim u. Basel 1986.

Vogelsang, W.: Asymmetrische Wahrnehmungsstile. In: Zeitschrift für Soziologie Sozialisationsforschung und der Erziehung, 20. Jg. (2000); Heft 3, S. 273-290.

Vollbrecht, R.: Die Herausforderung der Medienforschung angesichts gesellschaftlicher Modernisierungsprozesse. In: Deutsches Jugendinstitut (Hrsg.): Medien im Alltag von Kindern und Jugendlichen. München 1989.

Wallraff, L.: Alles, was rechts ist. In: die tageszeitung, 4.03.2001, S. 6.

Wensierski, H.-J.: Jugendsozialisation in ostdeutschen Jugendcliquen. In: Der pädagogische Blick, 8. Jg. (2000), Heft 4, S. 197-211.

Wiesner, C./Silbereisen, R. K.: Lebenslaufereignisse und biographische Muster in Kindheit und Jugend. In: Silbereisen, R. K./Vaskovics, L. A./Zinnecker, J. (Hrsg.): Jungsein in Deutschland. Jugendliche und junge Erwachsene 1991 und 1996. Opladen 1996, S. 185-198.

Wolsing, Th.: Die Kommerzialisierung von Kindheit und Jugend. In: deutsche jugend, 39. Jg. (1991), Heft 4, S. 175-186.

You-Team-Messe: outfit - sport - lifestyle. Bonn 2001.

Ziehe, Th.: Jugend, Alltagskultur und Fremdheiten. In: Negt, O. (Hrsg.): Die zweite Gesellschaftsreform. Göttingen 1994, S. 258-275.

Zinnecker, J.: Jugendkultur 1940 - 1985. Opladen 1987.

Zinnecker, J.: Pädagogische Ethnographie. In: Zeitschrift für Erziehungswissenschaft, 3. Jg. (2000[a]), Heft 3, S. 381-400.

Zinnecker, J.: Selbstsozialisation - Essay über ein aktuelles Konzept. In: Zeitschrift für Sozialisationsforschung und Soziologie der Erziehung, 20. Jg. (2000[b]); Heft 2, S. 181-202.

Zinnecker, J./Silbereisen, R. K.: Kindheit in Deutschland. Weinheim u. München 1998.

\section{Zum Autor}

Thole, Werner, Dr. phil. habil., Dipl.-Pädagoge und Dipl.-Sozialpädagoge; Professor für Jugend- und Erwachsenenbildung am Fachbereich Sozialwesen der Universität Kassel; Arbeitsschwerpunkte: Jugend und Kindheit, Kinder- und Jugendhilfe, insbesondere der außerschulischen Pädagogik mit Kindern und Jugendlichen, Theorie, Professionalisierungs-, Kindheits- und Jugendforschung, Theorie und Praxis der Sozialpädagogik.. 